\title{
A Tie2-Notch1 signaling axis regulates regeneration of the endothelial bone marrow niche
}

Lijian Shao, ${ }^{1}$ Kilian Sottoriva, ${ }^{1}$ Karol Palasiewicz, ${ }^{1}$ Jizhou Zhang, ${ }^{2}$ James Hyun, ${ }^{1}$ Sweta S. Soni, ${ }^{1} \mathrm{Na}$ Yoon Paik, ${ }^{1}$ Xiaopei Gao, ${ }^{1}$ Henar Cuervo, ${ }^{3}$ Asrar B. Malik, ${ }^{1}$ Jalees Rehman, ${ }^{1}$ Daniel Lucas ${ }^{2,4}$ and Kostandin V. Pajcini ${ }^{1}$

Haematologica 2019

Volume 104(11):2164-2177

${ }^{1}$ Department of Pharmacology, The University of Illinois College of Medicine, Chicago, IL 2Division of Experimental Hematology and Cancer Biology, Cincinnati Children's Medical Center, Cincinnati, $\mathrm{OH} ;{ }^{3}$ Department of Physiology and Biophysics, The University of Illinois College of Medicine, Chicago, IL and ${ }^{4}$ Department of Pediatrics, University of Cincinnati College of Medicine, Cincinnati, OH, USA

\section{ABSTRACT}

ૉ oss-of-function studies have determined that Notch signaling is essential for hematopoietic and endothelial development. By deleting a single allele of the Notch1 transcriptional activation domain we generated viable, post-natal mice exhibiting hypomorphic Notch signaling. These heterozygous mice, which lack only one copy of the transcriptional activation domain, appear normal and have no endothelial or hematopoietic phenotype, apart from an inherent, cell-autonomous defect in T-cell lineage development. Following chemotherapy, these hypomorphs exhibited severe pancytopenia, weight loss and morbidity. This phenotype was confirmed in an endothelial-specific, loss-of-function Notch1 model system. Ang1, secreted by hematopoietic progenitors after damage, activated endothelial Tie2 signaling, which in turn enhanced expression of Notch ligands and potentiated Notch1 receptor activation. In our heterozygous, hypomorphic model system, the mutant protein that lacks the Notch1 transcriptional activation domain accumulated in endothelial cells and interfered with optimal activity of the wildtype Notch1 transcriptional complex. Failure of the hypomorphic mutant to efficiently drive transcription of key gene targets such as Hes 1 and Myc prolonged apoptosis and limited regeneration of the bone marrow niche. Thus, basal Notch1 signaling is sufficient for niche development, but robust Notch activity is required for regeneration of the bone marrow endothelial niche and hematopoietic recovery.

doi:10.3324/haematol.2018.208660

Check the online version for the most updated information on this article, online supplements, and information on authorship \& disclosures: www.haematologica.org/content/104/11/2164

(C)2019 Ferrata Storti Foundation

Material published in Haematologica is covered by copyright. All rights are reserved to the Ferrata Storti Foundation. Use of published material is allowed under the following terms and conditions:

https://creativecommons.org/licenses/by-nc/4.0/legalcode. Copies of published material are allowed for personal or internal use. Sharing published material for non-commercial purposes is subject to the following conditions:

https://creativecommons.org/licenses/by-nc/4.0/legalcode, sect. 3. Reproducing and sharing published material for commercial purposes is not allowed without permission in writing from the publisher.

\section{Introduction}

Chemotherapy and radiotherapy are widely used in the treatment of hematopoietic malignancies but broad cytotoxicity is an undesirable feature of these treatments. ${ }^{1}$ These therapies damage multiple tissues including the bone marrow (BM) microvasculature. ${ }^{2.4}$ The regeneration of the endothelial $\mathrm{BM}$ vascular niche is crucial for successful reconstitution of hematopoietic cells. ${ }^{5,6}$ The interplay between the vascular and hematopoietic systems has multiple physiological and therapeutic implications. Endothelial cell (EC)-secreted growth factors such as vascular endothelial growth factor (VEGF)-A, enhance self-renewal and survival of hematopoietic stem cells (HSC) and mediate recovery of hematopoiesis. $5,7,8$ Angiopoietin-1 (Ang1) signaling, ${ }^{9,10}$ via the activation of tyrosine kinase Tie2 has been proposed as the key endocrine mechanism mediating endothelial recovery and regeneration. ${ }^{2,11}$ It is unknown whether paracrine signaling plays a role in the regeneration and reassembly of the BM endothelium.

Notch receptors are evolutionarily conserved transmembrane glycoproteins. Upon paracrine activation by neighboring cells through ligand interactions and proteolytic cleavage, they activate a transcriptional apparatus. ${ }^{12}$ Notch 1 and Notch 4 
are highly expressed in the endothelium during embryonic development and control EC specification ${ }^{13}$ and Notch1/Dl14 in coordination with VEGF-A/VEGFR2 signaling regulates sprouting angiogenesis. ${ }^{14,15}$ The function of Notch signaling in the adult vasculature is less understood. Studies showed that Notch1 signaling in the adult endothelium regulates expression of inflammatory genes. ${ }^{16}$ Notch1 is also known to be activated by blood flow and shear stress forces, which contribute to vascular homeostasis. ${ }^{17}$ Important, unresolved questions are whether Notch activation has a role in post-injury endothelial regeneration and whether it promotes the recovery of hematopoiesis.

The intracellular domains of Notch receptors have distinct roles. The RAM domain has a high affinity for binding to RBPJ, while the Ankyrin repeat (ANK) domains interact with a Mastermind-like (MAML) protein factor and recruit other co-activators. The PEST domain localized at the Cterminal facilitates Notch degradation. ${ }^{18}$ In between the ANK and PEST domains there is a transcriptional activation domain (TAD), which is capable of autonomous transcriptional activity and directly binds co-activators PCAF and GNC5. ${ }^{19,20}$ The TAD is a region of significant divergence among the four mammalian Notch receptors. ${ }^{20,21}$ These differences among the Notch receptor TAD may be important in the tissue-specific variability of Notch signaling.

We previously developed a transgenic knock-in model system which deleted the TAD of Notch1.22 This model system was used to study the role of Notch1 TAD function during fetal development. The loss of TAD in both Notch 1 alleles ( $\triangle T A D / \triangle T A D)$, allowed mice to develop to late gestation when they succumbed to multiple Notchdependent cardiovascular anomalies. ${ }^{22-24}$ While definitive HSC emerged from the aorta-gonad-mesonephros region, they failed to expand in the fetal liver of $\triangle T A D / \triangle T A D$ embryos. Furthermore, when transplanted into irradiated adult recipients, $\triangle T A D / \triangle T A D$ HSC underperformed in primary transplants and failed to reconstitute the hematopoietic system efficiently in secondary transplants..$^{22}$ In contrast, mice heterozygous for one allele of Notch1 $\triangle T A D\left(\right.$ Notch $\left.^{+/ \Delta T A D}\right)$ survive to adulthood and exhibit no overt hematopoietic phenotype.

In the present study, we made use of hypomorphic Notch signaling in the Notch $1^{+/ \Delta T A D}$ model to address whether the Notch pathway is crucial for the recovery of the adult BM niche and regeneration of hematopoietic cells after injury. We observed that high levels of Notch signaling were dispensable for the development of the endothelial niche and high Notch activity was not required during adult $\mathrm{BM}$ endothelial homeostasis. In the hematopoietic system, Notch $1^{+/ \Delta T A D}$ only displayed cellautonomous defects in the development of the T-cell lineage. However, following myelosuppressive injury, robust Notch signaling was critical for recovery of the BM endothelial niche and thereby the regeneration of HSC. Notch signaling was stimulated by a burst of Tie2-dependent activation, which induced expression of Notch1 ligands. Interestingly, expression of Notch $1 \Delta T A D$ protein in Notch $1^{+\Delta T A D}$ EC decreased expression of Notch target genes and led to severe apoptosis. This phenotype could not be rescued by enhanced activation of Tie 2 signaling. Our results suggest a crucial role for TAD-regulated Notch activity in mediating EC survival and promoting recovery of hematopoiesis following chemotherapeutic stress.

\section{Methods}

\section{Animals}

The following strains of mice were used in our studies under the guidelines and protocols approved by the Institutional Animal Care and Use Committees of University of Illinois at Chicago: C57BL/6J (or CD45.2), B6.SJL-Ptprc ${ }^{a} P$ ep 3 /BoyJ (or CD45.1),

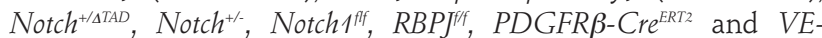
Cadherin $\mathrm{Cre}^{\mathrm{ERT}}$ mice. More details about the mice can be found in the Online Supplementary Methods.

\section{5-Fluorouracil treatment and irradiation}

5 -Fluorouracil (5-FU, $150 \mathrm{mg} / \mathrm{kg}$ ) was peritoneally injected into mice. For irradiation treatment, mice were exposed, on a rotating platform, to a lethal dose $(9.0 \mathrm{~Gy})$ of total body irradiation in a Mark I ${ }^{137} \mathrm{Ce} \gamma$-irradiator (JL Shepherd, Glendale, CA, USA) at a dose rate of $6.38 \mathrm{~Gy} / \mathrm{min}$. More details about chemotherapy and radiotherapy can be found in the Online Supplementary Methods.

\section{Analysis of bone marrow and thymic mononuclear cells}

Mononuclear cells from BM and thymus were prepared as described in the Online Supplementary Methods. The frequencies of LSK cells (Lin-Sca1 ${ }^{+} \mathrm{c}-\mathrm{kit}^{+}$cells), HSC (CD150+CD48-LSK cells), common lymphoid progenitors (CLP: LinSca1 ${ }^{\text {low }} \mathrm{C}$ kit ${ }^{\text {low }} \mathrm{CD} 135^{+} \mathrm{CD} 127^{+}$), thymic early T-cell precursors (ETP: Lin ${ }^{-}$kit ${ }^{+} \mathrm{CD} 25^{-\mathrm{CD}} 44^{+}$) and double-negative 3 cells (DN3 cells: Lin CD4-CD8 $\left.\mathrm{CD} 25^{+} \mathrm{CD} 44\right)$ were analyzed with a flow cytometer, Fortessa LSRII, after immunostaining with appropriate antibodies as described in the Online Supplementary Methods. All antibodies used are listed in Online Supplementary Table S1.

\section{Isolation of CD25+ thymocytes and primary bone endothelial cells}

Cell suspensions from the thymus or digested bones were incubated with dynabeads labeled with CD25 antibody and CD31 antibody (Invitrogen), respectively, for $30 \mathrm{~min}$ at $4^{\circ} \mathrm{C}$. $\mathrm{CD} 25^{+}$thymocytes and bone $\mathrm{CD} 31^{+}$cells (pBEC) were isolated and used for western blot and quantitative reverse transcriptase polymerase chain reaction (RT-qPCR) analysis.

\section{Bone marrow-derived endothelial cell culture}

Cultured bone marrow-derived endothelial cells (cBEC) were purchased from Cell Biologicals Inc (cat\#, C57-6221) and cultured in EC medium. cBEC were treated with a $\gamma$-secretase inhibitor (1 $\mu \mathrm{M}$, Sigma), a Tie2 kinase inhibitor (1 $\mu \mathrm{M}, \mathrm{Abcam})$, Ang1 (300 ng/mL, Peprotech) or 5-FU (100 $\mu \mathrm{M}$, Sigma). More detailed information can be found in the Online Supplementary Methods.

\section{Western blot analysis}

Expression of cleaved/active Notch1 (Val1744), Notch1, VECadherin, Tie2, and phosphorylated Tie2-Y992 was measured by western blot. The procedure is detailed in the Online Supplementary Methods.

\section{Quantitative reverse transcriptase polymerse chain reaction}

Expression of various genes was measured by RT-qPCR and calculated using the the comparative $\mathrm{C}_{\mathrm{T}}$ method. The sequences of all primers used in the RT-qPCR assay are listed in Online Supplementary Table S2.

\section{Chromatin immunoprecipitation assays}

RBPJ binding sites at Myc NDME, Hes1, Hey1 and Dtx1 locus 
were analyzed by chromatin immunoprecipitation assay. The procedure is described in detail in the Online Supplement. The sequences of primers are provided in Online Supplementary Table S3.

\section{Analysis of hematopoietic stem cell transplantation, apoptosis, luciferase activity, bone micro-computed tomography, sternum vasculature whole-mount imaging and immunostaining}

These assays and methods are described in the Online Supplementary Methods.

\section{Statistical analysis}

Details of the statistical analysis of the data are provided in the Online Supplementary Methods.

\section{Results}

\section{Myelosuppression by 5-fluorouracil or $\gamma$-irradiation causes pancytopenia in Notch $1^{+/ \triangle T A D}$ mice}

Myelosuppression by 5-FU is achieved by incorporating an analog of uracil into RNA or DNA of proliferating cells. ${ }^{25}$ The result of 5 -FU treatment during hematopoiesis is apoptosis of proliferating progenitors, followed by activation of HSC and reconstitution of the hematopoietic system. ${ }^{26}$ To test for a possible hematopoietic defect in Notch $1^{+/ \triangle T A D}$ mice, 5-week old wildtype (WT) and Notch $1^{+\Delta T A D}$ littermates were treated with two doses of 5FU, 14 days apart. Terminal hematopoietic analysis was performed 28 days after the first injection (Figure 1A). The condition of the Notch $1^{+/ \Delta T A D}$ mice deteriorated rapidly after treatment. Analysis of peripheral blood from Notch1 ${ }^{+/ \triangle T A D}$ mice revealed a sharp decrease in red blood cell and platelet counts, as well as hemoglobin concentration (Figure 1D). White blood cell and lymphocyte counts were also reduced in Notch $1^{+/ \Delta T A D}$ mice (Online Supplementary Figure S1A). During the first 9 days after treatment, all Notch $1^{+/ T A D}$ mice exhibited severe pancytopenia. For half of the Notch $1^{+/ \Delta T A D}$ mice, the body-score condition index and body weight dropped below humane levels (body condition index $=2$ ) (Figure $1 \mathrm{~B}, \mathrm{C})$, and the animals were sacrificed.

We analyzed bone marrow LSK (Lin $\left.-\mathrm{Sca}^{+} \mathrm{cKit}^{+}\right)$progenitors and HSC $\left(\mathrm{LSK}^{+} \mathrm{CD} 48 \mathrm{CD}^{-} 150^{+}\right)$after 5-FU treatment. Notch $1^{+/ T A D}$ mice showed significant decreases in the numbers of BM progenitors and HSC (Figure 1E) 9 days after treatment. The BM-residing common lymphoid progenitors (CLP), thymic early T-cell precursors (ETP) and CD4 CD8 double negative 3 (DN3) T-cell progenitors were also significantly reduced in Notch $1^{+/ \Delta T A D}$ mice after treatment (Figure 1F-H). Thymus size was reduced in 5-FU-treated Notch $1^{+\Delta T A D}$ mice compared to controls (Figure 1I). These findings indicate that TAD-dependent Notch1 signaling plays a critical role in recovery of the hematopoietic system following myelosuppression.

To determine whether ${ }^{137} \mathrm{Cs} \gamma$ total body irradiation had a similar effect as 5-FU treatment, WT or Notch $1^{1 / \Delta T A D}$ recipient mice $\left(\mathrm{CD} 45.2^{+}\right)$were lethally irradiated and subsequently transplanted with $2.0 \times 10^{6} \mathrm{WT}$ donor (CD45.1 ${ }^{+}$) BM cells. Recipient mice were monitored for 90 days after irradiation/transplantation (Online Supplementary Figure $S 2 A)$. We observed that the Notch $1^{+/ \Delta T A D}$ mice suffered severe weight loss (data not shown) and $50 \%$ of them had low body-score condition leading to mortality 9 days following irradiation/transplantation (Online Supplementary
Figure S2B). Total body irradiation has a limited effect on enucleated erythroid cells ${ }^{1}$ (Online Supplementary Figure S2C) but the numbers of white blood cells, neutrophils and lymphocytes were significantly lower in Notch $1^{+/ \Delta T A D}$ mice than in controls (Online Supplementary Figure S1B). At the 9-day time point, the numbers of donor-derived HSC, ETP, and DN3 cells in the Notch ${ }^{+/ \Delta T A D}$ recipient mice were markedly reduced (Online Supplementary Figure S2D), suggesting that the Notch $1^{+/ \Delta T A D}$ BM microenvironment plays a critical role during hematopoietic recovery. However, 90 days after transplantation, the numbers of the donorderived HSC, ETP, and DN3 populations were comparable between recipients (Online Supplementary Figure S2E) suggesting that in the surviving Notch $1^{+/ \Delta T A D}$ recipients, hematopoietic homeostasis was normalized. Thus, early hematopoietic recovery following $\gamma$ irradiation also depends on Notch1-TAD signaling.

\section{Transplantation with Notch1 $1^{\text {/ } \triangle T A D}$ hematopoietic stem cells reveals a cell-autonomous defect in T-cell development}

We next investigated whether there was a pre-existing hematopoietic defect in Notch1 $1^{+/ \Delta T A D}$ mice. Neither progenitors nor HSC were significantly affected in 6-week old Notch $1^{+/ \Delta T A D}$ animals (Online Supplementary Figure S3A). No effect was seen in marginal zone B cells in the spleen or in B- and T-cell populations in the lymph nodes in Notch $1^{+/ \Delta T A D}$ mice (Online Supplementary Figure S3B, C). The presence of a single Notch1- $\triangle T A D$ allele significantly reduced the frequency and number of thymic ETP and DN3 populations (Online Supplementary Figure S3D, E). This T-cell lineage defect could be traced to a reduction in the BM-residing CLP population (Online Supplementary Figure S3F). These findings show an inherent early role for Notch1-TAD signaling in the BM lymphoid progenitor population.

To investigate whether the effect of Notch $1^{+/ \Delta T A D}$ on hematopoietic recovery is cell autonomous, 350 HSC

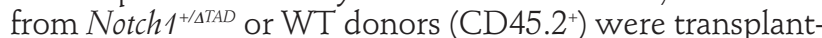
ed into congenic $\left(\mathrm{CD} 45.1^{+} / 2^{+}\right)$recipients (Figure $2 \mathrm{~A}$ ). Reconstitution, defined by having $85-90 \%$ of blood cells in the recipients derived from Notch $1^{+/ \triangle T A D}$ or WT donors, and multi-lineage potential was confirmed at 1, 2, and 3 months after transplantation (Online Supplementary Figure S4A). Notch $1^{+/ \Delta T A D}$ HSC reconstituted recipients similarly to WT HSC. There were equal numbers of donor-derived LSK cells and HSC in recipient BM (Online Supplementary Figure S4B), indicating that Notch $1^{+/ \triangle T A D}$ HSC were capable of homing and successfully engrafting into the WT recipient niche. In secondary transplants, mice receiving Notch $1^{+/ \Delta T A D}$ BM cells had no defects in HSC reconstitution (Online Supplementary Figure S4C, D); however, the CLP, ETP, and DN3 populations were significantly reduced (Online Supplementary Figure S4E, F). This finding indicated an inherent T-cell defect resulting from loss of one Notch1 $T A D$ allele but also showed that HSC reconstitution was otherwise unaffected.

To test the effects of chemotherapy on the Notch $1^{+/ \Delta T A D}$ hematopoietic system, we treated WT recipient mice reconstituted by Notch $1^{+/ \Delta T A D}$ HSC with two rounds of 5-FU (Figure 2A). Recipients transplanted with Notch $1^{+/ \Delta T A D}$ HSC exhibited no adverse effects when compared to those that received WT HSC (Figure $2 \mathrm{~B}$ ). Following treatment, comparable numbers of BM HSC were present in recipient mice (Figure 2C). Both sets of recipients had equivalent platelet, white blood cell, neutrophil, lymphocyte and red 
blood cell counts as well as hemoglobin levels (Online Supplementary Figure S4G). 5-FU treatment continued to affect the recovery of the T-cell lineage populations originating from Notch1 $1^{+/ \Delta T A D}$ donors (Figure 2D-F), which led to a $50 \%$ decrease in thymic mass (Figure $2 \mathrm{G}$ ). Our findings showed that loss of a single Notch 1 TAD allele markedly impaired T-cell development in the reconstituted WT recipients, but had no other adverse effects on hematopoiesis.

\section{Notch1 haploinsufficiency has no effect on} hematopoietic recovery

To test whether Notch1 haploinsufficiency can account for pancytopenia, weight loss and morbidity after chemotherapy, we treated Notch1 ${ }^{+/}$mice with 5-FU (Online Supplementary Figure S5A). Notch $1^{+/}$mice exhibited no significant difference in body score index compared to WT mice and had no discernable hematopoietic phenotype (Online Supplementary Figure S5B, C). Furthermore, no dif-

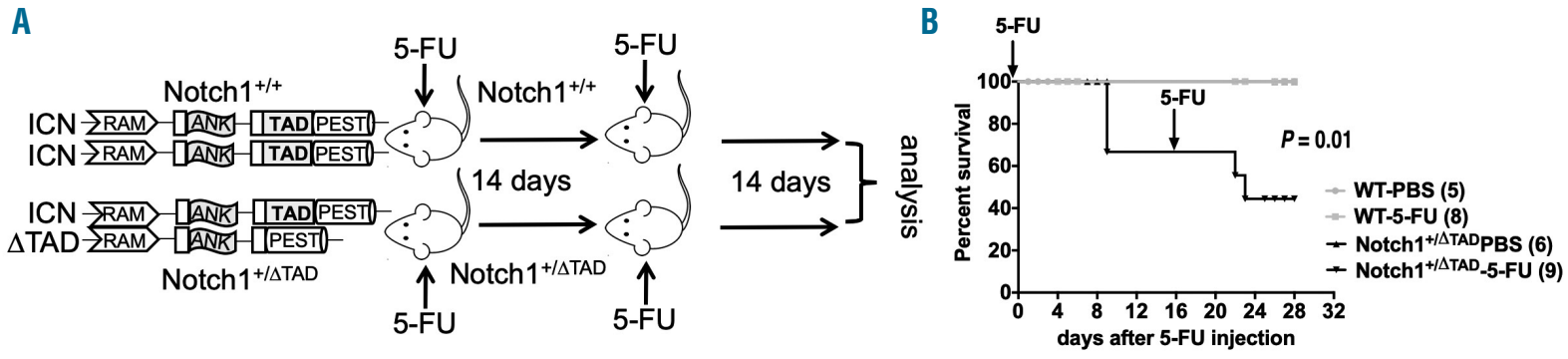

C

D
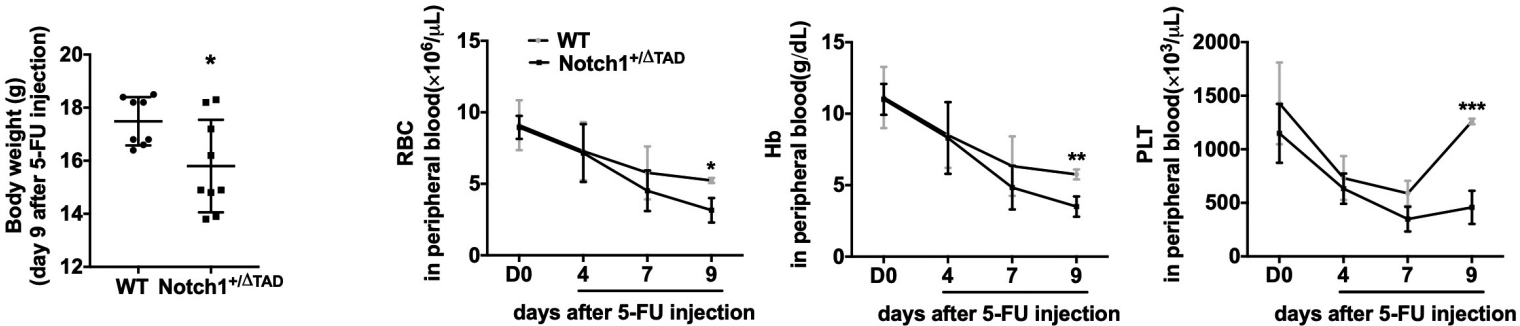

E

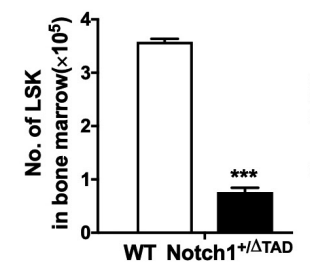

G

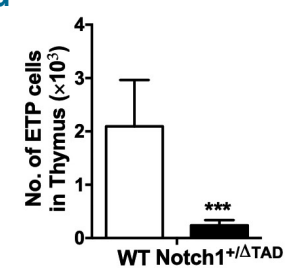

F
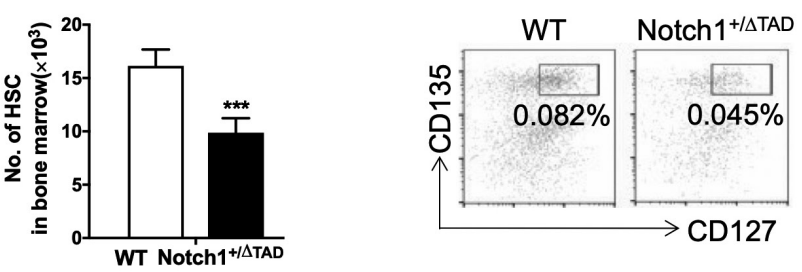

H

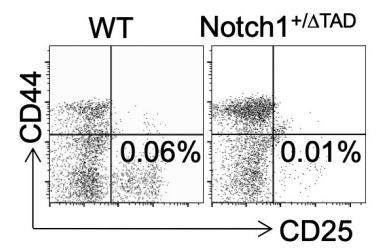

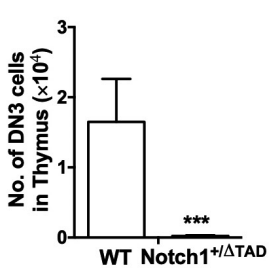

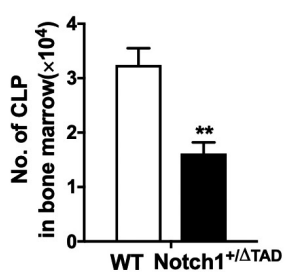

|

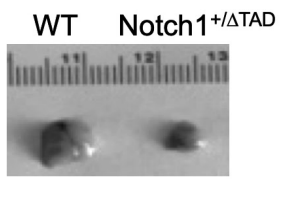

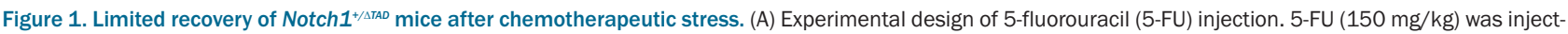
ed intraperioneally into Notch $1^{+/+}(W T)$ and Notch $1^{+\triangle A A D} 5$-week old mice on days 1 and 14 . (B) Kaplan-Meier plot indicating survival of WT and Notch $1^{+/ \Delta A A D}$ mice after 5-FU injection. Mice ( $n=5-9)$ were monitored daily until terminal analysis at day 28 after the first 5-FU injection. The statistical significance ( $P=0.01)$ between $W T$ and Notch $1^{+/ \Delta T A D} 5$-FU-treated littermates was determined by the Mantel-Cox test. (C) Body weight of WT and Notch $1^{+/ \Delta T A D}$ mice 9 days after 5-FU injection. (D) Red blood cell (RBC) and platelet (PLT) counts and hemoglobin ( $\mathrm{Hb}$ ) concentration were determined from peripheral blood of WT and Notch $1^{+/ \Delta T A D}$ mice ( $\mathrm{n}=5-9$ mice/group) before (DO) and at days 4,7 and 9 after 5 -FU injection. (E) Numbers of hematopoietic stem cells (HSC) and Lin Sca $1^{+} \mathrm{C}$ kit ${ }^{+}$(LSK) progenitors in the bone marrow were analyzed 9 days after 5 -FU injection. HSC were defined as Linc kit ${ }^{+}$Sca1 $1^{+} \mathrm{CD} 150^{+} \mathrm{CD} 48$ cells. The numbers of each population are expressed as mean \pm standard deviation (SD). (F) Cytometric analysis of common lymphoid progenitors (CLP) in bone marrow from WT and Notch1 $1^{+\Delta A A D}$ mice at day 14 after 5-FU injection. CLP were defined as Lin c-kit low Sca $1^{\text {low }} \mathrm{CD} 135^{+} \mathrm{CD} 127^{+}$(left panel). Absolute numbers of CLP from each mouse are expressed as mean \pm SD (right panel). (G, H) Cytometric analysis of early T-cell precursors (ETP) and double-negative 3 (DN3) cells in the thymus from WT and Notch1/_tAD mice at day 14 after 5-FU injection. ETP and DN3 populations were defined as Linc-kit ${ }^{+} \mathrm{CD} 25^{+} \mathrm{CD} 144^{+}(\mathrm{G})$ and LinCD4CD8CD44CD25+ $(\mathrm{H})$, respectively. Absolute numbers of ETP and DN3 cells from each mouse are expressed as mean \pm SD. (I) Representative images of the thymus from WT and Notch $1^{+/ \Delta T A D}$ mice at day 14 after 5 -FU injection. $* P<0.05, * * P<0.01, * * * P<0.001$. ICN: intracellular domains of Notch; TAD: transcriptional activation domain; RAM: RBP-J-associated molecule domain; ANK: ankyrin repeats. 
ferences were observed between WT and Notch1 ${ }^{+/}$littermates in hematopoietic progenitors and lineages in the BM and thymus (Online Supplementary Figure S5D-H). These findings show that a single allele of the Notch1 receptor was sufficient for recovery of the hematopoietic system and development of the T-cell lineage following myelosuppression. Thus, the phenotype observed in Notch ${ }^{+/ \Delta T A D}$ mice was unique and depended on the function of the TAD.

A
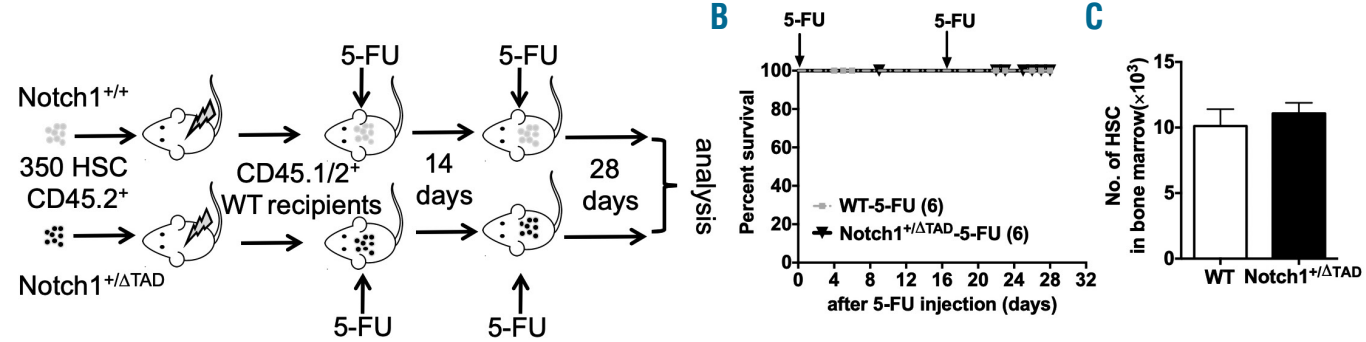

D

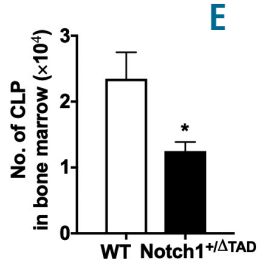

E

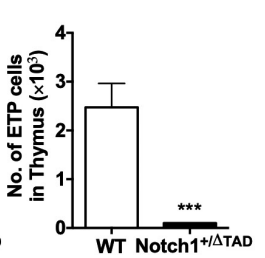

$\mathbf{F}$

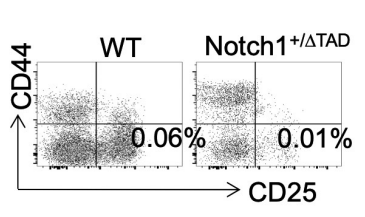

G
PBS

H

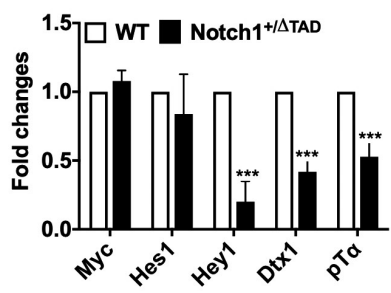

I

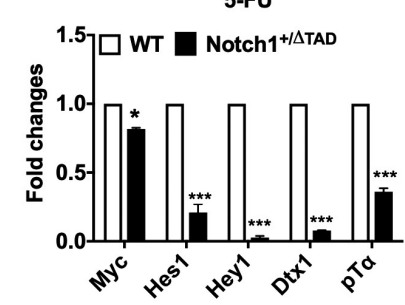

J

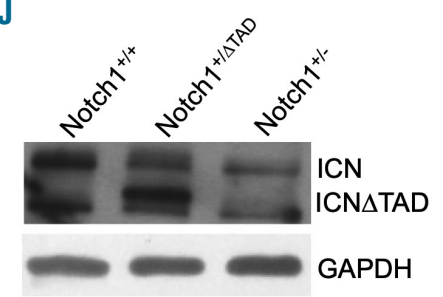

K

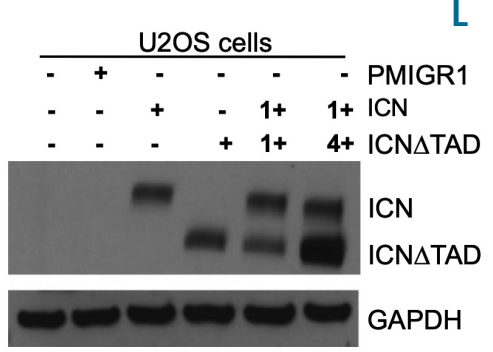

L

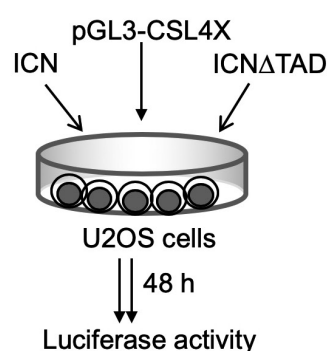

M

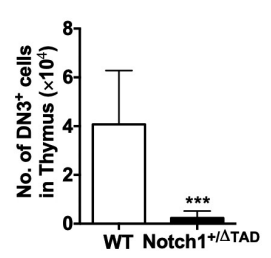

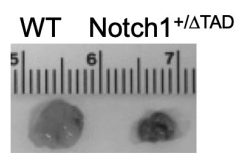

Figure 2. Transcriptional defect in T-cell development induced by Notch1 $1^{+/ \Delta T A D}$. (A) Experimental design of 5-fluorouracil (5-FU) treatment of recipient mice reconstituted with hematopoietic stem cells (HSC) from Notch $1^{+/ \triangle T A D}$ mice. Three hundred and fifty HSC from 5-week old wildtype (WT) or Notch $1^{+/ \triangle A A D}$ donors were sorted and transplanted into irradiated CD45.1/2+ WT recipients. After transplantation, 5-FU (150 mg/kg) was injected intraperioneally into recipient mice on days 1 and 14 . (B) Kaplan-Meier plot after 5-FU injection of recipients reconstituted by either WT or Notch $1^{+/ \Delta A D}$ HSC. Mice ( $\mathrm{n}=6 /$ group) were monitored daily until day 28 after the first 5-FU injection. Statistical significance was determined using the Mantel-Cox test. (C) Absolute numbers of donor-derived HSC in the bone marrow 14 days after the 5-FU injection. (D) Absolute numbers of donor-derived common lymphoid progenitors (CLP) in bone marrow at day 14 after the 5-FU injection are shown as mean \pm standard deviation (SD). (E, F) Cytometric analysis and quantification of donor-derived early T-cell precursors (ETP) and double-negative 3 (DN3) cells from the thymus of reconstituted mice at day 14 after 5-FU injection. Absolute numbers are shown as mean \pm SD. (G) Representative images of the thymus from recipient mice at day 14 after 5-FU injection. $(\mathrm{H}, \mathrm{I})$ Expression of the indicated genes in the DN3 population from WT or Notch $1^{+/ \Delta T A}$ mice $(n=3)$. Thymic DN3 cells collected by flow-activated cell sorting at day 9 after injection of phosphate-buffered saline (PBS) (H) or 5-FU (I). Fold changes in the relative gene expression in Notch $1^{+/ \Delta T A D}$ DN3 cells are shown normalized to WT expression and GAPDH. (J) Expression of Notch1-ICN and Notch1-ICN $A T A D$ in CD25+ thymocytes ( $n=3$ ). CD25+ thymocytes from WT, Notch $1^{+/ \Delta T A D}$ and $\mathrm{Notch}^{+/}$mice were isolated by anti-CD25 dynabead magnetic sorting and whole cells were lysed to measure protein expression of cleaved Notch1 ICN (val1744) by western blot. ICN is $100 \mathrm{kDa}$, ICN $\triangle T A D$ is $75 \mathrm{kDa}$, and a non-specific band of $\sim 65 \mathrm{kDa}$ is seen in all three lanes. (K) U2OS cells transfected with pMIGR1 control vector, pMIGR1-Notch1 ICN and pMIGR1-Notch1 ICN $\triangle T$ TAD. At $48 \mathrm{~h}$, protein expression of Notch1 ICN and Notch1 ICN $\triangle T A D$ was measured by western blot. (L) Experimental design of the luciferase assay. U20S cells were transfected with reporters pRL-TK, pGL3-CSL4X, and with either pMIGR1 control vector or ICN or ICN $\triangle$ TAD or both ICN and ICN $\triangle T A D$ at indicated ratios $(n=3)$. (M) Firefly luciferase activity was measured relative to renilla luciferase activity. Values are shown relative to the empty vector. $* P<0.05, * * P<0.01, * * * P<0.001$. 


\section{Transcriptional defect in Notch1 $1^{+/ \triangle T A D}$ impairs T-cell} development

Notch function in T-cell development has been well documented, ${ }^{27,28}$ so to determine how lack of a single TAD allele affects $T$ cells, we measured target expression in sorted WT and Notch $1^{1 / \Delta T A D}$ DN3 cells before and after 5-FU treatment. During resting conditions, no significant change was observed in the expression of Hes1 or Myc, but a decrease in the levels of Hey 1 and Dtx 1 was seen in

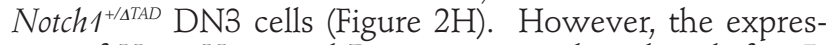
sion of Hes1, Hey1, and Dtx1 was severely reduced after 5FU treatment, and expression of pre-T-cell receptor alpha $(p T \alpha)$ and $M y c$ was moderately reduced (Figure 2I), suggesting that Notch $1 \triangle T A D$ strongly impairs target gene expression in developing thymocytes during regeneration.

To determine whether production of active Notch1 was equal among our different model systems, CD25 thymo-

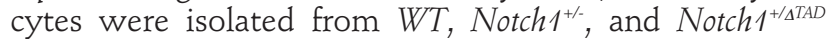
mice. The abundance of cleaved, full-length intracellular domains of Notch (ICN) was comparable between

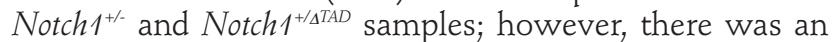
accumulation and a nearly 3 -fold increase of the levels of cleaved ICN $\triangle$ TAD protein (Figure 2J).

Next, we employed an in vitro U2OS-based Notch luciferase reporter assay to assess the effects of accumulated ICN $\triangle T A D$ on transcription. ${ }^{29}$ We chose U2OS cells, a human osteosarcoma cell line, because they express very low levels of endogenous Notch signaling and are highly transfectable. Constructs expressing Notch1 WT or $\triangle T A D$ mutant were transfected into U2OS cells at various ratios of ICN to ICN $\triangle T A D$ (Figure $2 \mathrm{~K}, \mathrm{~L}$ ). ICN $\Delta \mathrm{TAD}$ had a lower capacity of transcriptional activation compared to ICN (Figure 2M, lanes 3 and 4). Dose-dependent transcriptional inhibition was observed when ICNATAD was cotransfected with ICN. The presence of a 1:4 ratio of ICN:ICN $\triangle T A D$ caused a 4 -fold decrease in luciferase activity (Figure $2 \mathrm{M}$, lane 6). This TAD-dependent regiment was rescued by inverting the ratio of ICN to ICN $\Delta$ $\mathrm{TAD}$ (Figure $2 \mathrm{M}$, lane 7). Our findings uncovered a role for the $\triangle \mathrm{TAD}$-mediated suppression of WT Notch signaling which we termed TAD transcriptional interference. These findings showed that the presence of a Notch1 $\Delta \mathrm{TAD}$ protein impaired Notch target expression. Since Notch signaling is crucial for T-lineage development, we conclude that this transcriptional interference negatively affected in vivo development of thymocytes.

\section{Chemotherapy severely damages bone marrow microvasculature and induces endothelial cell apoptosis in Notch1 $1^{+/ \triangle A D}$ mice}

Our findings suggest that the cell-intrinsic hematopoietic defect in Notch1 ${ }^{+\Delta T A D}$ is limited to the T-cell lineage, which cannot account for the pancytopenia, morbidity, and the eventual mortality observed in the Notch $1^{+\triangle I A D}$ mice (Figures 1 and 2). Thus, our attention turned to the BM microenvironment. First, to elucidate whether loss of one copy of TAD in the Notch1 locus affects bone and mesenchymal cells, we performed micro-computed tomography analysis of femoral bones. This assay indicated comparable bone length, bone volume fraction (BV/TV) and cortical thickness in WT and Notch $1^{+/ A T A D}$ mice (Online Supplementary Figure S6A-C). Furthermore, CD51 and Sca1 were used to identify osteoblasts and mesenchymal stem/precursor cells. ${ }^{30}$ Flow cytometry analysis showed that there were similar numbers of osteoblasts and mesenchymal stem/precursor cells in digested bones from WT and Notch $1^{+\Delta T A D}$ mice (Online Supplementary Figure $S 6 D, E)$. These data indicate that loss of one copy of Notch1 TAD does not significantly affect bone development in Notch1 $1^{+/ A T D}$ mice.

To test whether the presence of one Notch1 $\triangle T A D$ allele affects formation of the bone vasculature network, wholemount sternal imaging was conducted. Imaging of the vasculature labeled by CD31 and VE-cadherin showed comparable vessel number and vessel length between $W T$ and Notch $1^{+/ \triangle T A D}$ mice under resting conditions (Figure 3A). Next, we wanted to determine the effect of chemotherapy on the recovery and regeneration of BM EC and specifically the sinusoidal EC, which have been shown to be crucial components of the adult hematopoietic niche. ${ }^{31-33}$ Primary bone CD31+ EC (pBEC) were harvested from WT mice 3 days after 5-FU treatment. Expression of the Notch1 receptor and several Notch canonical gene targets were upregulated after the chemotherapy (Figure 3B). Target gene upregulation corresponded with Notch1 receptor cleavage (Figure 3B, inset), showing an increase in Notch activation which occurred in pBEC as early as 3 days after 5 -FU treatment.

To determine the condition of the BM EC niche at the time point when the Notch1 $\triangle T A D$-expressing heterozygous hypomorphs succumb to myelosuppressive treatment, pBEC in WT or Notch1 ${ }^{+\Delta T A D}$ littermates were analyzed 9 days after 5-FU treatment (Figure 3C). Annexin V staining showed that $20 \%$ of the $\mathrm{pBEC}$ in Notch $1^{+/ \Delta T A D}$ mice were apoptotic compared to $<7 \%$ of WT pBEC (Figure 3D). This sorted pBEC population from digested bones, over $90 \%$ of which expressed CD $31^{+}$and less than $5 \%$ expressed $\mathrm{CD}_{4} 5^{+}$(Figure 3E, left panel), was further analyzed for expression of pro-apoptotic genes. A corresponding 2-fold increase in the expression of Puma and Bax ${ }^{34}$ was observed in sorted Notch ${ }^{+/ \Delta T A D}$ pBEC (Figure 3E, right panel). Absolute numbers of Notch1 ${ }^{+/ \Delta T A D}$ pBEC were markedly decreased, reflecting apoptosis (Figure 3F).

To visualize the $\mathrm{BM}$ microenvironment, ${ }^{35}$ sections of the tibia of WT and Notch1 $1^{+/ \Delta T A D}$ littermates were analyzed before and after chemotherapy. Under resting conditions, as suggested by the whole mount imaging of the sternum (Figure 3A), there was no difference in the abundance of hematopoietic cells in the $\mathrm{BM}$, and similar $\mathrm{CD} 31^{+}$and endomucin ${ }^{+}$sinusoidal endothelial structures were present in WT and Notch1 ${ }^{+/ \Delta T A D}$ littermates (Figure 3G-I, top panels). After 5-FU treatment, retention of hematopoietic cells was markedly reduced in Notch1 $1^{+/ A T A D}$ BM (Figure 3G, bottom panel). Severe damage to the BM niche following 5-FU treatment was evident by the lack of $\mathrm{CD} 1^{+}$cells and irregular endomucin ${ }^{+}$endothelial staining in the Notch $1^{+/ \triangle A D}$ mice following this treatment (Figure 3H-I, bottom panel). Overall, our findings indicate that the hematopoietic defect observed in Notch1 $1^{+/ \Delta T A D}$ mice can be attributed to the failure of endothelial regeneration after chemotherapy.

\section{Endothelial cell-specific deletion of Notch1 receptor confirms the requirement for endothelial Notch signaling during niche recovery}

To specify a tissue-specific role for Notch signaling in the recovery and regeneration of BM EC, Notch ff $^{\text {VE-cadherin- }}$ $\mathrm{Cre}^{\mathrm{ERT} T 2+}$ mice were treated with tamoxifen 1 week prior to administration of 5-FU (Figure 4A). The tamoxifen regiment led to loss of Notch1 protein (Figure 4B) and a 
decrease in Notch target expression (Figure 4C) in pBEC. The condition of Notch ${ }^{f f}$ VE-cadherin-Cre ${ }^{E R T 2+}$ mice deteriorated (body condition index $=2$ ) within a week after treatment, and five of 13 mice succumbed between days 7-9 after treatment (Figure 4D). The morbidity of Notch ${ }^{f f} V E-$ cadherin-Cre $e^{E R T 2+}$ mice was reflected in the low peripheral blood counts (Figure 4E) as well as decreases in the numbers of BM HSC, progenitors and CLP (Figure 4F, G). Consistent with increased endothelial apoptosis after treatment, the absolute number of pBEC in Notch ${ }^{f f} V E$-cadherin$\mathrm{Cre}^{\text {ERT2+ }}$ mice was significantly reduced (Figure $\left.4 \mathrm{H}, \mathrm{I}\right)$.

Besides the endothelium, perivascular cells have been implicated in the regulation of HSC function. ${ }^{36}$ We observed a decrease in numbers of BM PDGFR $\beta^{+}$pericytes after chemotherapy in Notch $1^{+/ \Delta T A D}$ mice. However, this effect was not due to increased apoptosis of the pericytes and may be attributed to a previously described loss of
BM EC (Online Supplementary Figure S7A). To determine whether Notch signaling in pericytes played a role in the recovery of the BM niche, RBPJff-PDGFR $\beta$-Cre ${ }^{E R T 2+}$ and $R B P \int^{f+}-P D G F R \beta-C r e^{E R T 2+}$ littermates were treated first with tamoxifen and then with 5-FU (Online Supplementary Figure $S 7 B, C)$. We observed no significant hematopoietic differences between the two cohorts after 5-FU treatment (Online Supplementary Figure S7D-I). Overall these results show that robust Notch signaling in EC is essential for recovery of the $\mathrm{BM}$ niche following chemotherapeutic challenge.

\section{Loss of the transcriptional activation domain suppresses transcriptional activation of Notch1 targets in endothelial cells}

To determine whether the $\triangle \mathrm{TAD}$-dependent transcriptional interference observed in T cells also occurred EC
A
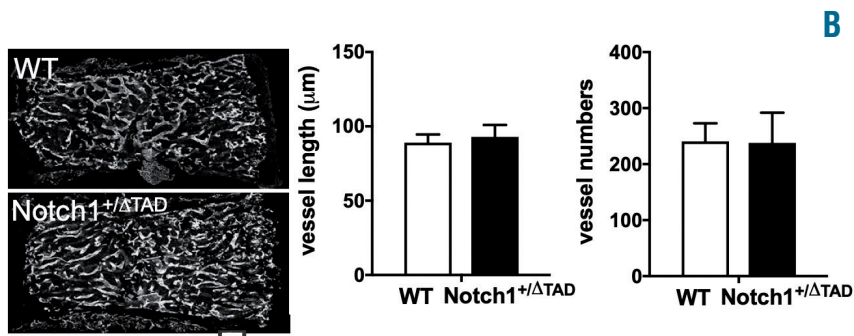

E

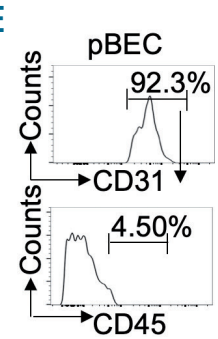

B

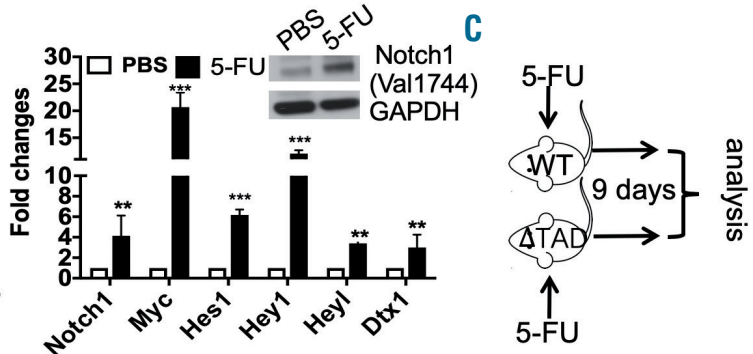

$F$
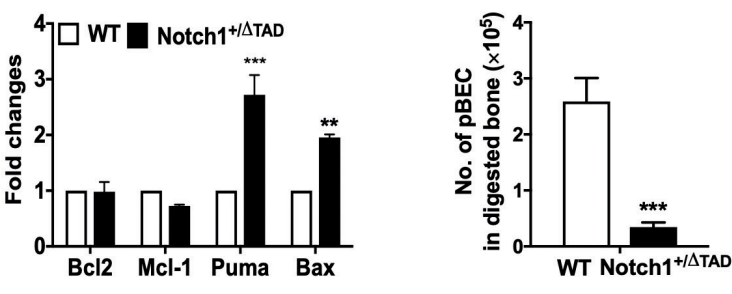

CD31
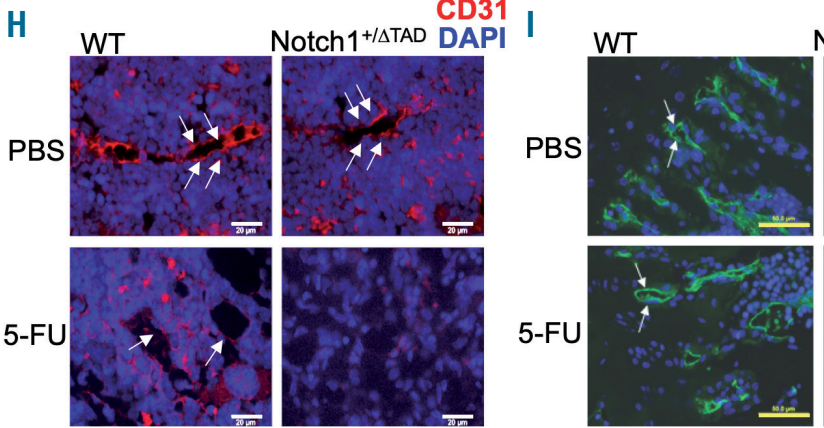

Endmc

Notch $1^{+/ \Delta T A D}$

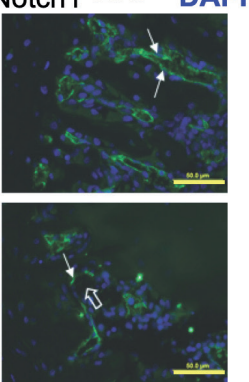

Figure 3. Defective recovery of endothelial niche induced by Notch1 $1^{+/ A A D}$. (A) Whole mount imaging of the sternal vasculature after staining for CD31 and VE-cadherin was performed in WT and Notch $1^{+\triangle A A D}$ mice under resting conditions, including analysis of vessel length and vessel numbers ( $\left.\mathrm{n}=4\right)$. Scale bar $=150 \mu \mathrm{m}$. (B) $\mathrm{CD} 31^{+}$primary bone endothelial cells (pBEC) were isolated at day 3 after 5 -fluorouracil (5-FU) injection and expression of the indicated genes was determined by quantitative reverse transciptase polymerase chain reaction (RT-qPCR) normalized to expression of genes after injection of phosphate-buffered saline (PBS) ( $\mathrm{n}=3$ ). GAPDH was used as an internal expression control. Levels of active Notch1 protein in pBEC were measured by western blot at day 3 after 5 -FU injection (insert). (C) Experimental design of 5-FU treatment for endothelial cell analysis in bone marrow $(n=6)$. (D) pBEC harvested from long bones of WT and Notch $1^{+/ \Delta T A D}$ littermates gated for 4',6-diamidino-2-phenylindole (DAPI)CD45 TER119'CD31 ${ }^{+}$and analyzed by flow cytometry for annexin V at day 9 after PBS or 5-FU injection (left panel). Percentages of annexin $\mathrm{V}^{+}$cells in $\mathrm{CD} 31^{+}$endothelial cells $(\mathrm{pBEC})$ are presented as mean $\pm \mathrm{SD}$ (right panel). (E) Isolated pBEC from digested bones were stained with DAPICD45 TER119 CD31 ${ }^{+}$. The purity of CD31 ${ }^{+}$cells as a percent of CD $31^{+} \mathrm{CD} 45^{+}$cells is demonstrated in the histogram analysis (left panel). The expression of indicated genes was determined by RT-qPCR in PBEC isolated from WT and Notch $1^{+/ \triangle A D}$ mice at day 7 after 5 -FU injection (right panel). Fold changes in the relative gene expression, normalized to WT samples with GAPDH used as an internal expression control, are shown as mean \pm SD. (F) Absolute numbers of pBEC (DAPICD45 TER119 $\mathrm{CD} 1^{+}$) were analyzed at day 9 after 5 -FU injection by flow cytometry. (G) Representative longitudinal bone sections from mice treated with PBS (upper panels) or 5-FU (lower panels) stained with hematoxylin and eosin-Y at day 9 after 5 -FU treatment. Scale bar $=50 \mu \mathrm{m}$. (H, I) Representative longitudinal bone section from mice treated with PBS (upper panels) or 5-FU (lower panel) fixed with formalin and stained for CD31 (H, red, scale bar=20 $\mu \mathrm{m})$ or endomucin (endmc) (I, green, scale bar $=100 \mu \mathrm{m}$ ) at day 9 after 5 -FU injection. DAPI was used to stain nuclei (blue). Filled white arrows point to vascular structures representative of the bone marrow endothelium. The open white arrow points to discontinuous vascular structures. $* * P<0.01, * * * P<0.001$. 
and whether it affected the recovery of EC in the BM niche, we tested the expression of Notch target genes in pBEC. Under basal conditions, expression of Hes1, Hey1 and Dtx1 was downregulated in Notch $1^{+/ \Delta T A D}$ pBEC, while expression of $M y c$ and EphB2 was unaffected (Figure 5A). However, 9 days after 5-FU treatment, expression of the
Notch target genes EphB2, Myc, Hes1, Hey1 and Dtx1 was markedly downregulated in Notch ${ }^{+/ \Delta A A D}$ pBEC (Figure 5B). To determine how Notch target expression was affected by the Notch $1 \Delta \mathrm{TAD}$ mutant, a chromatin immunoprecipitation assay was performed on purified pBEC from WT or Notch $1^{+\triangle T A D}$ littermates after 5-FU treatment. The chromatin
A

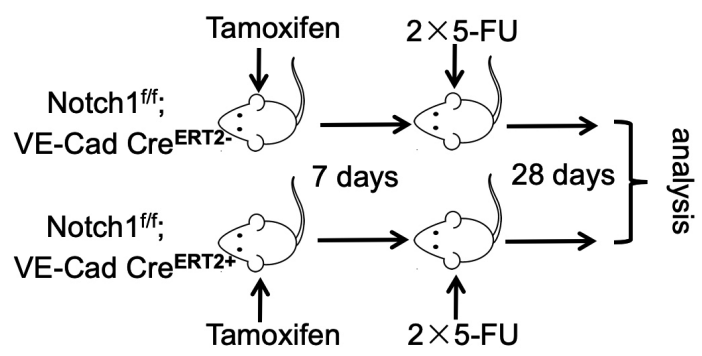

C

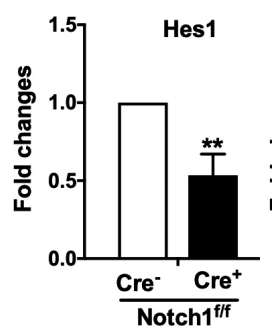

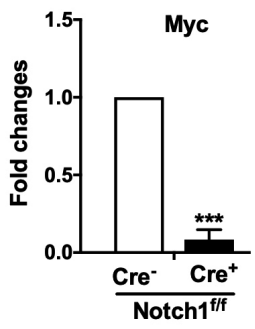

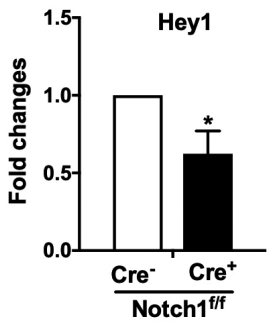

B

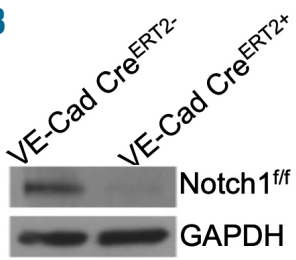

D

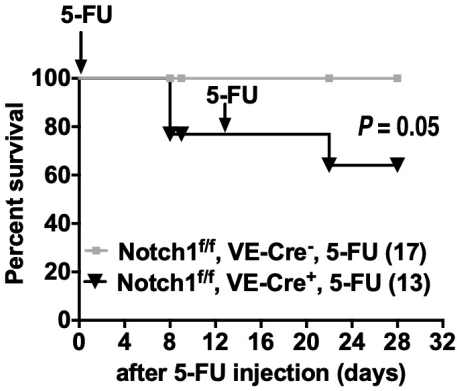

$\mathrm{E}$
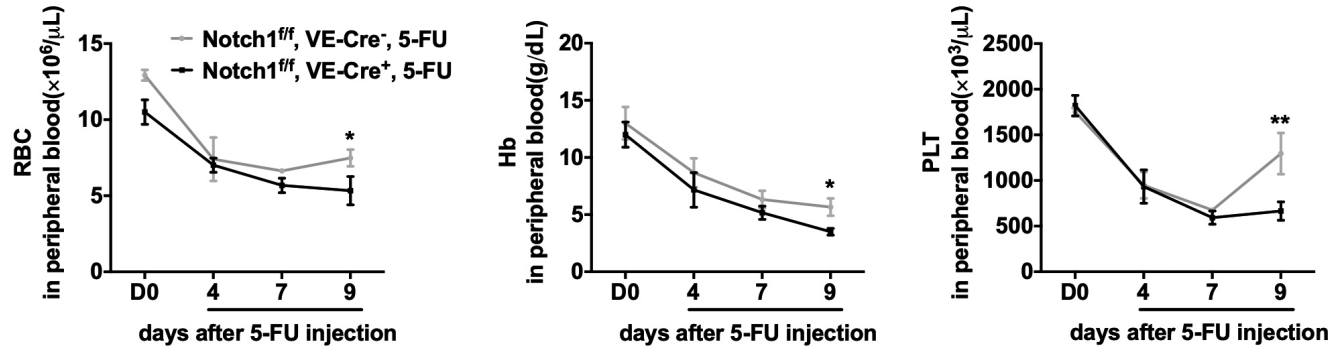

$\mathbf{F}$
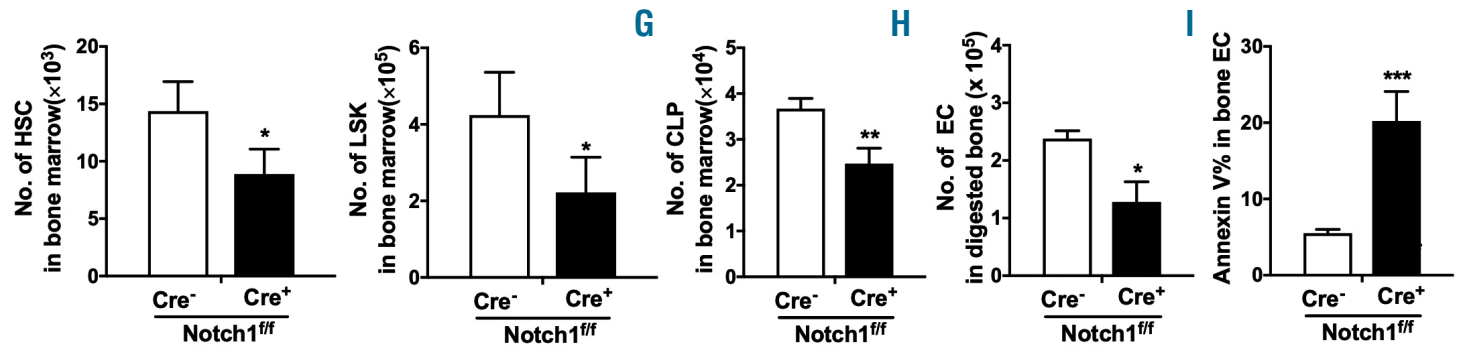

Figure 4. Conditional deletion of Notch1 in endothelial cells phenocopies Notch1 $1^{+/ \Delta A D}$ mice after myelosuppression. (A) Experimental design of 5-fluorouracil (5-

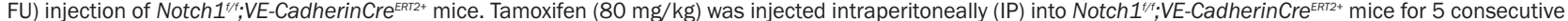

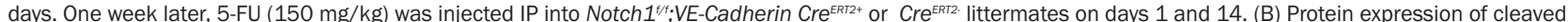
Notch1 in $\mathrm{CD} 1^{+}$primary bone endothelial cells (pBEC) from Notch $1^{t / f}$; VE-Cadherin Cre ${ }^{\text {ERT2 }}$ or Cre ${ }^{\text {ERT2+ }}$ littermates after tamoxifen treatment. GAPDH was used as a loading control. (C) Quantitative reverse transcriptase polymerase chain reaction (RT-qPCR) expression of Hes1, Myc, Hey1 and Dtx1 in pBEC harvested from

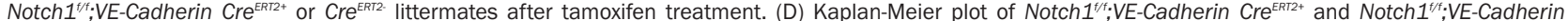
$\mathrm{Cre}^{\text {ERT2. }}$ littermates after tamoxifen and 5-FU treatment. Mice were monitored daily until day 28 after 5-FU injection ( $\left.\mathrm{n}=13-17 \mathrm{mice} / \mathrm{group}\right)$. Significance (P=0.05) was determined by the Mantel-Cox test. (E) Peripheral blood counts of red blood cells (RBC) and platelets (PLT), and concentration of hemoglobin (Hb) in Notch $1^{t / f} ; \mathrm{VE}$-CadherinCre ${ }^{E R T 2+}$ and Cre ${ }^{\text {ERT2 }}$.littermates were conducted before (DO) and at days 4,7 and 9 after 5-FU injection. (F) Absolute numbers of hematopoietic stem cells (HSC) and Lin Sca1 ${ }^{+} \mathrm{c}-\mathrm{kit}^{+}$(LSK) cells in bone marrow were analyzed 9 days after the 5-FU injection and expressed as mean \pm standard deviation (SD). (G) Absolute numbers of common lymphoid progenitors (CLP) in bone marrow were analyzed at day 9 after the 5-FU injection and expressed as mean \pm SD. (H) Absolute numbers of pBEC were analyzed at day 9 after the 5 -FU injection by flow cytometry. (I) Percentages of annexin $\mathrm{V}^{+}$Lin-Sca1 ${ }^{+} \mathrm{C}-\mathrm{Kit} \mathrm{t}^{+} \mathrm{pBEC} 9$ days after 5 -FU injection are presented as mean \pm SD. ${ }^{*} P<0.05, * * P<0.01, * * * P<0.001$. 
immunoprecipitation assay showed reduction of Notch1 occupancy at the Notch-dependent Myc enhancer NDME ${ }^{37}$ and at Hes1, Hey1 and Dtx1 promoters ${ }^{38}$ in Notch ${ }^{+/ \Delta T A D}$ pBEC (Figure 6C). In line with our findings in thymocytes (Figure 2J), we observed a 3-fold accumulation of Notch1 ICN $\triangle T A D$ protein in $\mathrm{pBEC}$ when compared to cleaved Notch1 WT protein (Figure 5D). These results indicate that $\triangle \mathrm{TAD}$ interference occurred in $\mathrm{pBEC}$ and contributed to the decreased target gene expression in EC, thus limiting regeneration and recovery of the BM niche.
For further in vitro study of $\triangle \mathrm{TAD}$ transcriptional interference, $\mathrm{pBEC}$ were sorted from the BM by expression of $\mathrm{CD}_{3} 1^{+}$and VE-cadherin ${ }^{+}$(Online Supplementary Figure $S 8 A)$. These cultured bone EC (cBEC) exhibited typical endothelial morphology, VE-cadherin ${ }^{+}$adherent junctions and active Notch signaling (Online Supplementary Figure $S 8 B, C)$. To replicate the effects of $\triangle \mathrm{TAD}$ interference, we transduced cBEC with a retroviral vector expressing GFP (PMIGR1) or ICNDTAD (Figure 5E, left). Accumulation of ICN $\triangle \mathrm{TAD}$ protein in $\mathrm{GFP}^{+}$sorted $\mathrm{cBEC}$
A

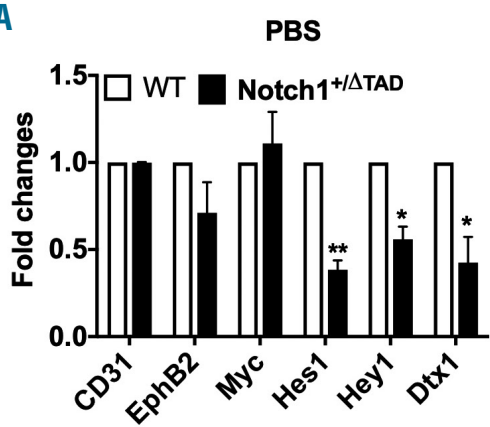

B

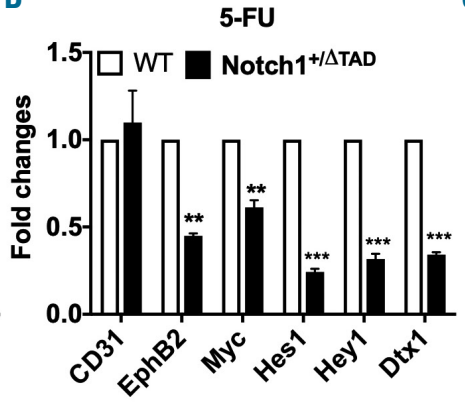

C

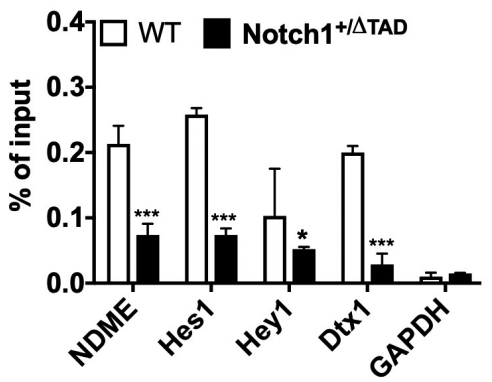

D

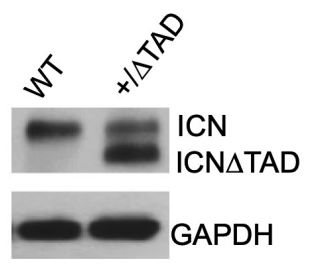

F

F Veh

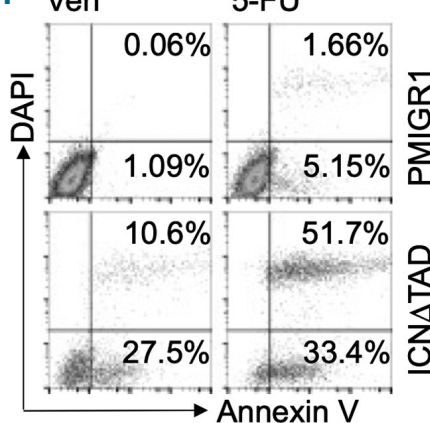

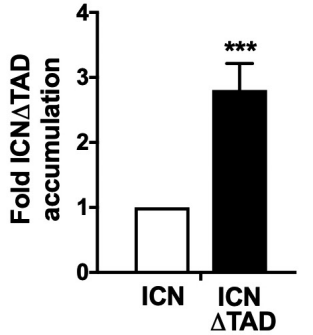

G
E

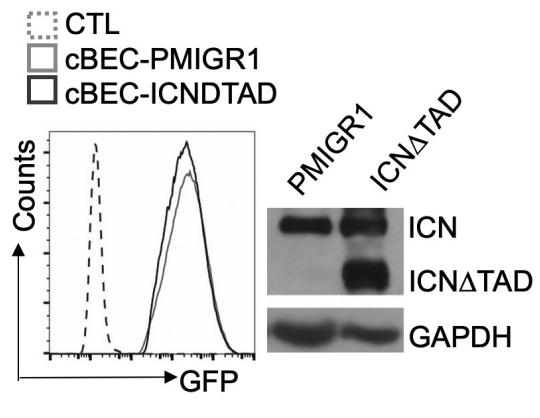

H

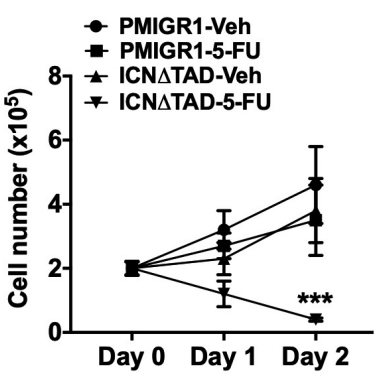

I

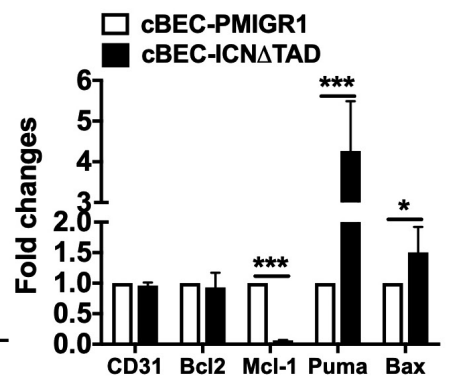

Figure 5. Notch1 ICN $\triangle T$ TAD interference in bone marrow endothelial cells. (A, B) The expression of the indicated genes was determined by quantitative reverse transcriptase polymerase chain reaction (RT-qPCR) in CD31 primary bone endothelial cells (pBEC) at day 9 after treatment of wildtype (WT) and Notch $1^{+/ \Delta T A D}$ littermates $(n=3)$ with phosphate-buffered saline (PBS) or 5-fluorouracil (5-FU). Values were normalized to expression of genes in WT littermates. GAPDH was used as an internal expression control. (C) Local chromatin immunoprecipitation (CHIP) assay to detect the occupancy of Notch1 complex on Rbpj DNA-binding sites of Notch-dependent Myc enhancer (NDME), Hes1, Hey1, Dtx1 and GAPDH promoter elements. pBEC from WT and Notch1 ${ }^{+/ \Delta T A D}$ mice were used for CHIP analysis at day 9 after 5-FU treatment $(n=3)$. Values represent the mean of signal intensity relative to input DNA normalized to IgG. (D) pBEC from WT and Notch1 ${ }^{+/ \Delta T A D}$ mice were harvested and used to measure protein levels of cleaved Notch1 and Notch1 $\triangle T A D$ by western blot (left panel). The band intensities of Notch1 ICN and Notch1 ICN $\triangle T A D$ in Notch1 $1^{+\triangle T A D}$ pBEC were quantified by Image J software ( $n=3$, right panel). ( $E$ ) Flow cytometry analysis of cultured bone marrow-derived endothelial cells (cBEC) (untransduced control) or cBEC transduced with retrovirus expressing PMIGR1 or PMIGR1-ICN $\triangle$ TAD (left panel). Western blot analysis for expression of Notch1 protein in transduced and sorted GFP ${ }^{+}$CBEC expressing PMIGR1 or PMIGR1-ICN $\triangle$ TAD. GAPDH was used as a loading control ( $n=3$, right panel). (F, G) cBEC transduced with PMIGR1 or PMIGR1-ICN $\triangle T$ TAD were treated with 5-FU or vehicle (Veh) for $24 \mathrm{~h}(\mathrm{n}=3)$. Representative annexin $\mathrm{V}$ and 4',6-diamidino-2-phenylindole (DAPI) flow cytometry analysis $(\mathrm{F})$ and quantified percentages of pro-apoptotic annexin $\mathrm{V}^{+}$cells $(\mathrm{G})$. (H) Cell counts of cBEC-PMIGR1 and cBEC-ICN $\triangle$ TAD after Veh or 5-FU treatment. Cell numbers were counted at day 1 and day 2 after a single 5-FU treatment $(n=3)$. (I) RT-qPCR for the indicated genes in cBEC-PMIGR1 and cBEC-ICN $\triangle$ TAD cells $24 \mathrm{~h}$ after 5 -FU treatment. Values were normalized to PMIGR1 transduced cells. GAPDH was used as an internal expression control. $* P<0.05, * * P<0.01, * * * P<0.001$. 
was confirmed by western blot analysis (Figure 5E, right).

Increased ICN $\triangle T A D$ in $c B E C$ markedly impaired the expression of Hey 1 and Dtx 1 and significantly downregulated the expression of Myc (Online Supplementary Figure S8D). To mimic chemotherapy in vitro, cBEC were treated with $100 \mu \mathrm{M}$ of 5 -FU. After $24 \mathrm{~h}$, the cells were analyzed for apoptosis by flow cytometry. We observed that $27 \%$ of cBEC-ICN $\triangle \mathrm{TAD}$ cells were pro-apoptotic (annexin $\mathrm{V}^{+}$) under resting conditions; furthermore, the apoptotic cells (annexin $\mathrm{V}^{+} / \mathrm{DAPI}^{+}$) cells increased to $50 \%$ in 5 -FU-treated cBEC-ICN $\triangle T A D$ (Figure 5F, G). Apoptosis of cBEC-ICN $\Delta$ $\mathrm{TAD}$ led to a rapid decrease in cell numbers $48 \mathrm{~h}$ after 5 -FU treatment (Figure $5 \mathrm{H}$ ). This finding was validated by a decrease of Mcl-1 expression and an increase of Puma and Bax expression in cBEC-ICN $\triangle \mathrm{TAD}$ (Figure 5I). These findings corroborate the in vivo phenotype observed in freshly isolated BM EC and indicate the importance of Notch signaling in the recovery and survival of EC after chemotherapy.
Both Notch1 and Tie2 signaling are activated during recovery of bone marrow endothelium

Tie2 signaling has been proposed to facilitate endothelial regeneration., ${ }^{2,39}$ Thus, we compared the expression of the Notch 1 and Tie2 receptors 3, 5, and 7 days after 5-FU treatment in the pBEC of WT mice. Tie 2 expression was low in resting $\mathrm{pBEC}$ and rapidly increased by 10 -fold at day 5 after 5-FU treatment (Figure 6A). This increase in Tie2 expression corresponded with a significant increase in the phosphorylation of Tie2 protein (p-Tie2) (Figure 6B, right panel). Expression of Notch1 receptor was increased 4-fold after treatment, but Notch1 receptor expression and activation remained high throughout until day 7 (Figure 6A, B). Previous work has suggested that activation of Tie 2 signaling by Ang1 in human vascular EC results in increased induction of Notch ligand delta-like 4 (D114). ${ }^{40}$ To test this in vivo, we treated WT mice with 5-FU (Figure 6C). Five days later, expression of Dll4 and Jag1, determined by analysis of both mRNA (Figure 6D) and protein levels
A
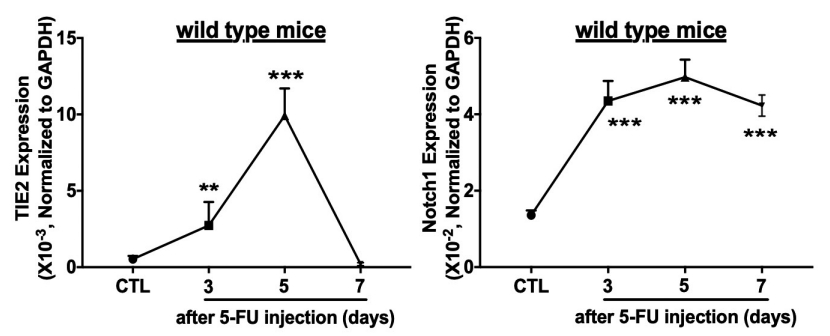

C

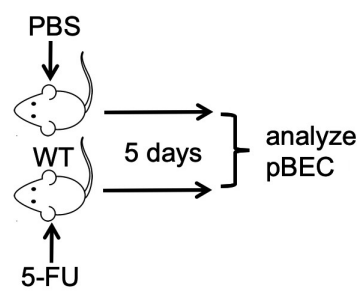

D

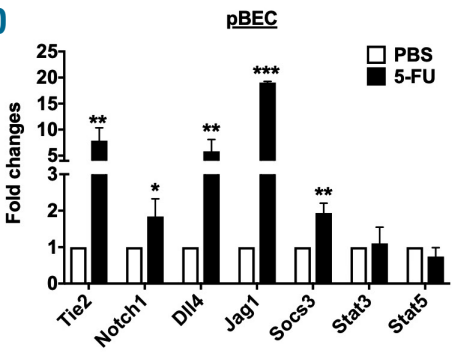

B

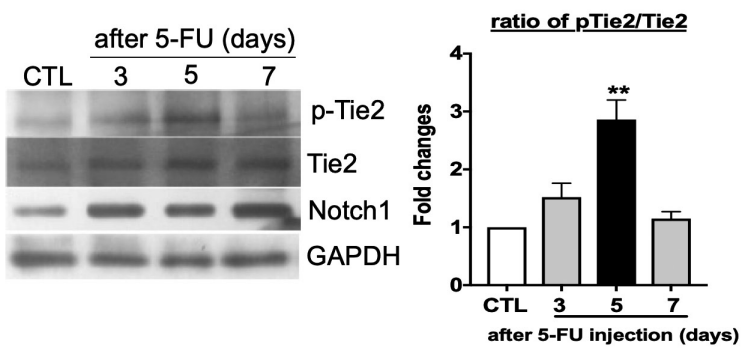

$E$
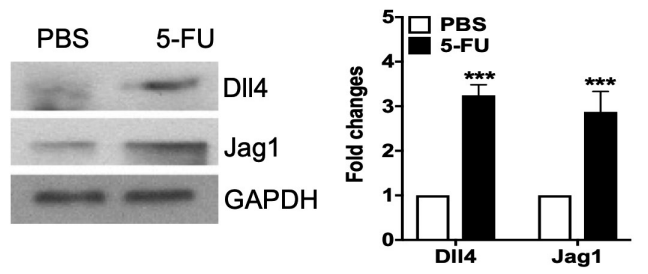

$\mathbf{F}$

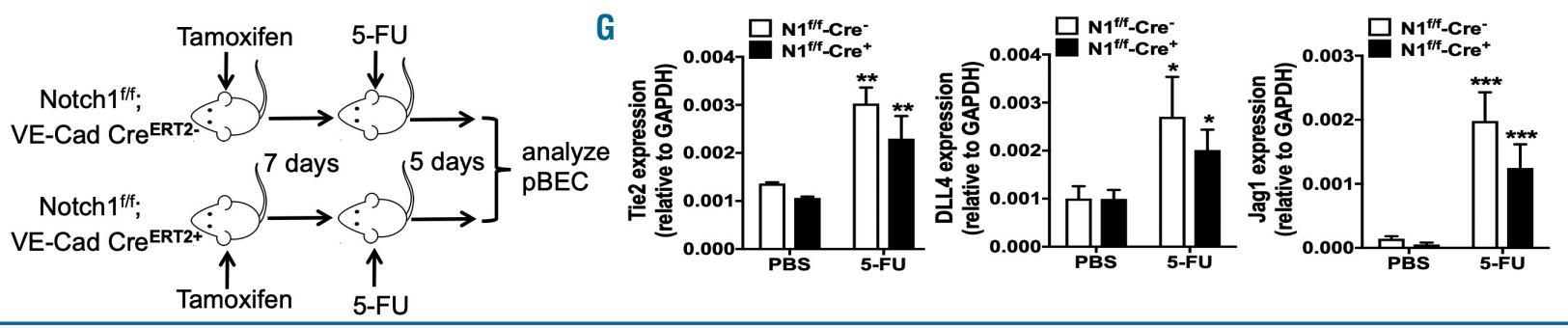

Figure 6. Both Notch1 and Tie2 signaling are activated during endothelial niche recovery after chemotherapy. (A) Expression kinetics of Tie2 and Notch1 after chemotherapy. Quantitative reverse transcriptase polymerase chain reaction (RT-qPCR) expression of Tie2 and Notch1 in sorted CD $31^{+}$primary bone endothelial cells (pBEC) from wildtype (WT) mice before (CTL) and at days 3, 5 and 7 after 5-fluorouracil (5-FU) treatment. GAPDH was used as an internal expression control at each time-point. (B) Representative western blot of protein level analysis for phosphorylated Tie2 (p-Tie2), total Tie2, cleaved Notch1 and GAPDH from sorted pBEC before (CTL) and at days 3, 5 and 7 after 5-FU treatment (left panel). The band intensities of Tie 2 and p-Tie 2 in pBEC were quantified by Image J software. The ratios of pTie2/Tie2 at days 3,5 and 7 after 5-FU treatment were normalized to that of the control (CTL) group. The fold changes of pTie2/Tie2 ratio are presented ( $n=3$, right panel). (C) Experimental design of 5-FU treatment for pBEC analysis (phosphate-buffered saline, PBS, n=6; 5-FU, n=15). Five- to six-week old mice were treated intraperitoneally (IP) with PBS or 5-FU. CD31+Ter119CD45 cells (pBEC) were sorted from digested bones 5 days after 5-FU treatment. (D) RT-qPCR expression of indicated genes from pBEC treated as described in (C). Values are normalized to PBS-treated pBEC. GAPDH was used as an internal expression control. (E) Protein expression of DII4 and Jag1 was measured in pBEC from mice treated with PBS or 5-FU. GAPDH was used as a loading control (left panel). The band intensities of

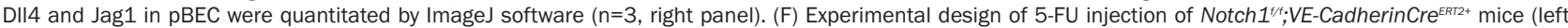
panel). Notch $1^{t / f} ; \mathrm{VE}$-CadherinCre ${ }^{\text {ERT2 }}$ mice were injected IP with tamoxifen daily for 5 days. One week later, 5-FU was injected IP into Notch $1^{t / f} ; \mathrm{VE}$-Cadherin CreERT2+ or CreERT2 littermates. pBEC were analyzed at day 5 after 5-FU injection ( $n=3$ ). (G) Expression of Tie2, DII4 and Jag1 was measured by RT-qPCR in pBEC as described in (F). Absolute expression values were compared to those of PBS-injected mice of each genotype. GAPDH was used as an internal expression control. 
(Figure 6E), was significantly increased in pBEC from both BM (Online Supplementary Figure S8E) and digested bone (Figure 6D, E). Notch ligand upregulation corresponded with the upregulation of Tie2, Notch1 and the Tie2 signaling target Socs3 (Figure 6D, E, Online Supplementary Figure S8E).

Our initial expression timeline (Figure 6A) indicated that Notch1 expression is upregulated prior to Tiez upregulation. To determine whether the induction of Tiez and Notch ligands after chemotherapy depended on active Notch signaling, we employed the previously described Notch $1^{\text {Pf } V E-c a d h e r i n-C r e ~}{ }^{\mathrm{ERT} 2+}$ model system and analyzed freshly harvested pBEC from WT (Cre) and Notch1'frVEcadherin-Cre ${ }^{E R T 2+}\left(\mathrm{Cre}^{+}\right)$mice after tamoxifen and 5-FU treatment (Figure 6F). Our data indicate that 5-FU treatment significantly increased expression of Tie2, Dll4 and Jag1 in pBEC in WT and Notch 1f VE-cadherin-Cre ${ }^{\text {ERT2 }}$ mice. However, loss of endothelial Notch1 expression did not significantly affect upregulation of Tie2 after 5-FU treatment (Figure 6G, left panel). Furthermore, loss of Notch signaling did not significantly affect the expression of Notch ligands Dll4 and Jag1 (Figure 6G), upregulation of which coincided with the increase in Tiez expression and activity (Figure 6A, B). Taken together, we show that Tie2 signaling is independent of Notch1 signaling during BM endothelial regeneration and recovery.

\section{Notch1 functions downstream of Tie2 signaling during the recovery of bone marrow endothelium}

Previous studies have shown that hematopoietic progenitors and mesenchymal cells are responsible for the secretion of Ang1 in the BM. ${ }^{10}$ To test this, hematopoietic progenitor (Lin-Sca-1 $1^{+}$) cells and bone osteoblasts (CD51+) were sorted from the BM 5 days after 5-FU treatment. Expression of Ang1 was markedly increased in 5-FU-treated hematopoietic progenitors (up to 150-fold) and osteoblasts (up to 30-fold) when compared to the same untreated cell populations (Figure 7A). To further analyze any possible crosstalk between the Notch and Tie-2 signaling pathways, we supplemented cBEC culture media with Ang1, which caused an increase in the levels of $\mathrm{p}$ Tie2 and cleaved Notch1 (Figure 7B). Ang1 stimulation also induced the expression of Notch ligands (Dll4 and Jag1) and its targets, such as Hes1, Hey1 and Myc (Figure 7C). To determine whether Tie2 stimulation by Ang1 was essential for Notch ligand upregulation, we treated cBEC with a Tie2 inhibitor. Our results show that increased expression of Dll4 and Jag1 by Ang1 stimulation was completely blocked by pre-treatment with the Tie2 kinase inhibitor (Figure 7D). Our findings support a role for the Tie2 signaling pathway in the induction of Notch signaling by enhancing expression of Notch ligands.

To determine whether Notch signaling also influenced Tie2 expression, we inhibited Notch activity by addition of a $\gamma$ secretase inhibitor for $48 \mathrm{~h}$ followed by a $3 \mathrm{~h}$ washout. Notch targets, inhibited by $\gamma$ secretase inhibitors, are rapidly upregulated during washout. We observed that this was the case for Hes 1 in CBEC, but not for Tiez and Ang2 (Figure 7E). Interestingly, Myc expression in $\mathrm{CBEC}$ was not entirely dependent on Notch activity; however, $M y c$ levels markedly increased after the washout of the $\gamma$ secretase inhibitor, indicating that in EC $M y c$ is responsive to higher levels of Notch signaling (Figure 7E). These data showed that, in BM EC, Tie2 activation enhanced downstream Notch signaling while Notch activity had no effect on Tie2 signaling.
To test whether Notch functioned downstream of Tie2 activation, ICN $\triangle T A D-c B E C$ cells were treated with 5-FU for $24 \mathrm{~h}$ and then the medium was supplemented with Ang1 (Figure 7F, left). Ang1 stimulated moderate growth of resting $c B E C$ but had no effect on the ICN $\triangle T A D$-transduced cBEC. After 5-FU treatment, Ang1 significantly increased growth of control $c B E C$ but did not rescue ICN $\triangle T A D-c B E C$ (Figure 7F, right). Tie2 activation by Ang1 had the expected result of increasing Socs 3 expression. ${ }^{41}$ However, Tie- 2 activation had no effect on the Notch targets Hes 1 and Hey1 in ICN $\Delta \mathrm{TAD}$-expressing cells (Figure 7G). These results show that whereas Tie2 activation accelerates the recovery of the BM EC niche after chemotherapy by promoting Notch ligand expression, ultimately, the resolution of niche recovery depends on activation of robust Notch signaling.

\section{Discussion}

The Notch1 receptor is crucial for the emergence of the first definitive HSC from the embryonic hemangioblast ${ }^{42}$ and expansion of fetal liver HSC.22 The role of Notch signaling in adult BM HSC is not well understood and mired in controversy. ${ }^{43-46}$ It has been suggested that Notch signaling is essential for HSC self-renewal, specifically with regard to Jagged1 activation of Notch1 signaling in HSC. ${ }^{46}$ Even so, pan-inhibition of Notch signaling in HSC by deletion of the key transcriptional complex member and DNA-binding factor RBPJ, showed no adverse effects in maintenance and expansion of the stem and progenitor pool. ${ }^{47}$ A separate recent study showed depletion of HSC numbers after complete ablation of Notch signaling in the endothelium, thus suggesting that the Notch1 receptor participates in the development of the EC niche. ${ }^{48}$ An even more recent article indicated a possible alternative to Notch1 BM signaling by suggesting that Jagged2 triggers Notch2 signaling in HSC after myelosuppression. ${ }^{49}$

Regardless of its proposed involvement during hematopoietic and endothelial development, the specific role of Notch signaling, its mechanistic interplay with other known regenerative pathways and its temporal activation during and following myelosuppression are not known. In the present study, we investigated Notch signaling in adult hematopoietic and endothelial tissues by deleting the TAD from a single allele of Notch1. This mutation has the capacity to interfere with the activity of the WT Notch1 transcriptional complex and created a hypomorphic signaling environment. Our Notch1 ${ }^{+/ \Delta T A D}$ heterozygous model system matured to adulthood and under homeostatic conditions exhibited no adverse effects in the bone and vasculature. We show that this heterozygous hypomorphic model system is useful for determining the requirement for robust Notch signaling activation in adult tissues. Using the Notch1 $1^{+\Delta T A D}$ mice we determined that basal Notch1 signaling was sufficient for development of the endothelial hematopoietic niche; however, robust activation of Notch1 was required for the regeneration of the hematopoietic system.

We observed increased Notch1 receptor expression and cleavage in BM EC following treatment with the chemotherapeutic agent 5-FU. The WT BM microenvironment began to recover at $\sim 9$ days after exposure to 5 -FU. However, the BM niche was persistently disrupted in Notch $1^{+/ T A D}$ mice as evidenced by the presence of few CD31 
and endomucin ${ }^{+}$microvessels and a high frequency of apoptotic EC. Defective regeneration of EC in these mice caused gaps in sinusoidal vessels leading to severe hemorrhage. ${ }^{5,50}$ The occupancy of the Notch transcriptional complex and expression of Notch target genes associated with survival and proliferation, such as Hes1 and $M y c^{51,52}$ was markedly decreased in Notch $1^{+/ \triangle T A D}$ BM EC, indicating that the regeneration of niche EC following chemotherapy was dependent on high levels of Notch signaling. Validation was obtained using conditional deletion of the Notch1 receptor in the VE-cadherin ${ }^{+}$EC. Similar to the Notch $1^{+\Delta T A D}$, this ECspecific, conditional, loss-of-function Notch1 model exhibited defective regeneration of BM EC highlighted by increased EC apoptosis and limited hematopoietic recovery.
A

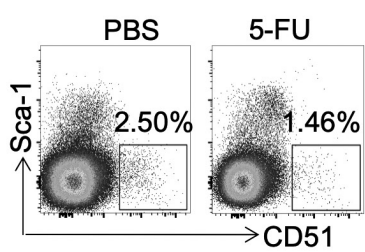

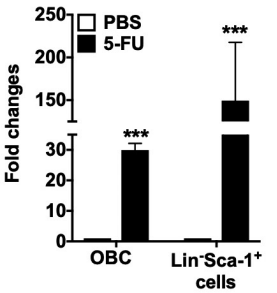

D

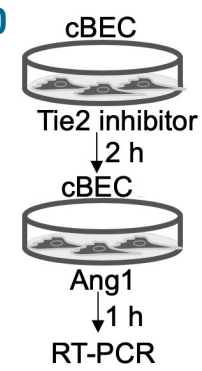

B

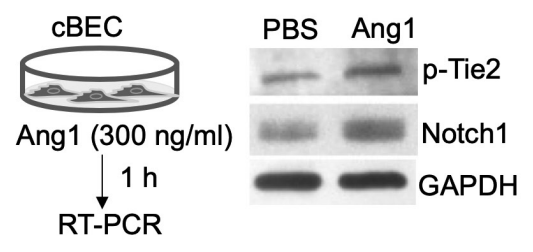

C

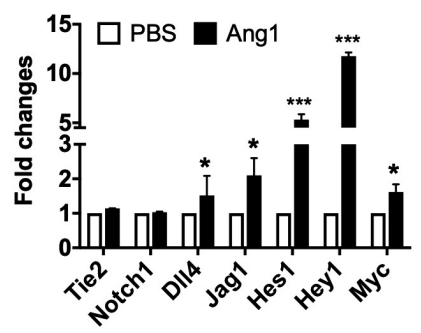

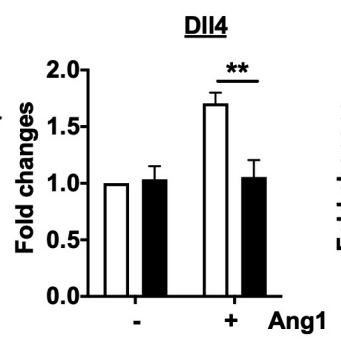

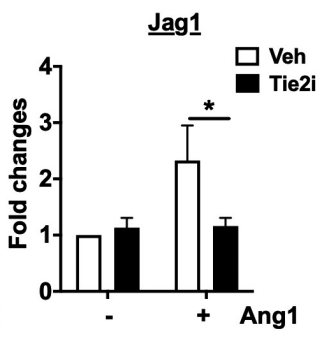

E

$\mathrm{F}$

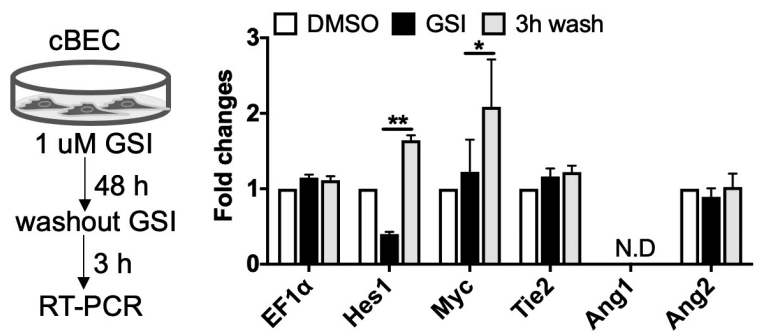

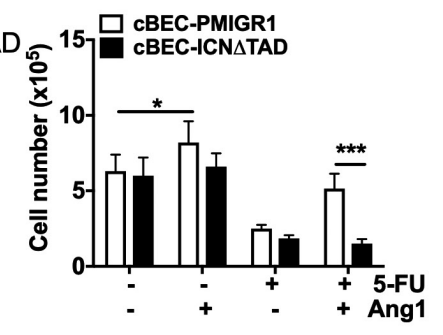

G
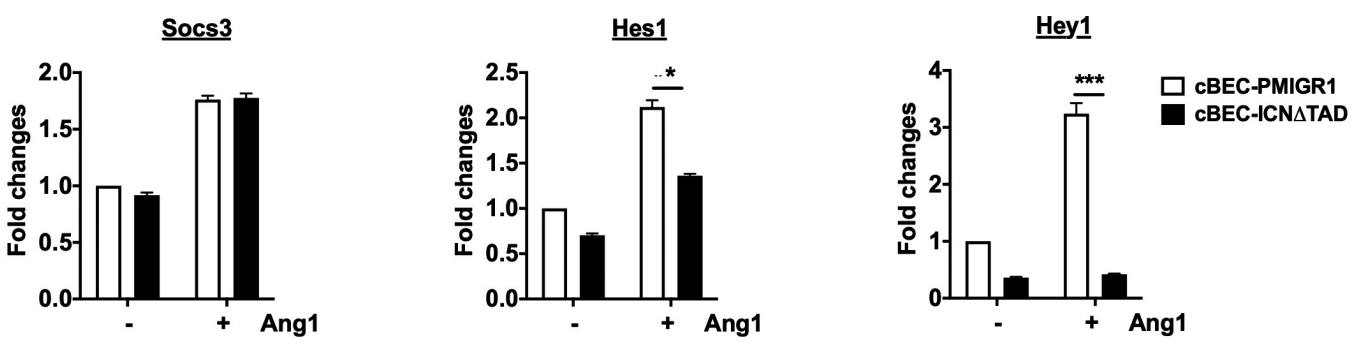

Figure 7. Notch1 functions downstream of Tie2 signaling to mediate endothelial niche recovery. (A) Representative gating strategy for osteoblasts (Ter119-CD45 CD31:Sca1 CD51 $^{+}$cells) in digested bones from mice treated with phosphate-buffered saline (PBS) and 5-fluorouracil (5-FU) (PBS, $n=5$; 5-FU, $n=15$; left panel). Expression of Ang1 was determined by quantitative reverse transcriptase polymerase chain reaction (RT-qPCR) in osteoblasts and Lin Sca-1 $1^{+}$cells treated with PBS and 5-FU (right panel). GAPDH was used as an internal expression control. (B) Schematic of Ang1 treatment in serum-starved (16 h) cultured bone marrow-derived endothelial cells (cBEC) (left panel). Expression of p-Tie2, cleaved Notch1 and GAPDH protein levels in Ang1-treated cBEC was detected by western blot (right panel). (C) RT-qPCR expression of Notch target genes from cBEC treated as described in (B). Values are normalized to those in PBS-treated cBEC. GAPDH was used as an internal expression control. (D) Schematic of Tie2 inhibitor (Tie2i) and Ang1 treatment in serum-starved (16 hr) cBEC (left panel). Expression of DIl4 and Jag1 was determined by RT-qPCR in Tie2i and/or Ang1-treated cBEC. The vehicle for the Tie2 inhibitor was dimethysulfoxide (DMSO). Vehicle for Ang1 is PBS. (E) Schematic for treatment of cBEC with $\gamma$ secretase inhibitors (GSI). RT-qPCR expression analysis of the indicated genes in cBEC treated with GSI/washout. Values were normalized to those of DMSO-treated cBEC. GAPDH was used as an internal expression control. (F) Schematic for treatment of control PMIGR1 or PMIGR1-ICNATAD cBEC with 5-FU and Ang1. The indicated cBEC populations of cells were monitored for growth after 5-FU and Ang1 treatment. (G) RT-qPCR expression of Tie2 target Socs3, and Notch targets Hes1 and Hey1 was examined after cell growth analysis of cBEC in (F). Values were normalized to those of PMIGR1 cBEC without Ang1-treatment. GAPDH was used as an internal expression control. Unless indicated differently, $\mathrm{n}=3$ were used for experiments in this figure. $* P<0.05, * \star P<0.01, * * * P<0.001$. 
While further work is required to determine whether specific subsets of BM EC are more susceptible to loss of Notch signaling during niche regeneration, our work suggests that a majority of EC, which express CD31, VE-cadherin and endomucin, require robust Notch1 signaling to recovery after myelosuppressive injury.

Mediators of BM niche recovery include VEGF-A, Tie2, and the Tie2 agonist Ang1., ${ }^{2,53,54}$ Initiation of regenerative signaling is believed to hinge upon VEGF-A produced by osteoblasts and osteocytes ${ }^{55,56}$ and Ang1 produced by osteoprogenitors and hematopoietic precursors. ${ }^{10,57} \mathrm{We}$ observed that there was a short pulse of Tie2 phosphorylation after chemotherapeutic damage while Notch signaling remained at elevated levels for at least 7 days after chemotherapy. Tie2 activation increased Notch receptor cleavage and the Tie 2 agonist, Ang1 stimulated Notch signaling and promoted the expression of two canonical Notch ligands: Dll4 and Jag1. However, Tie2 stimulation, after 5-FU treatment failed to rescue the survival of Notchdeficient EC which expressed the hypomorphic Notch $\triangle T A D$ allele. These results indicate that Notch signaling functions downstream of Tie 2 and that Notch1 signaling is ultimately responsible for the regeneration of the BM endothelium.

The Notch $1^{+\Delta T A D}$ mice exhibited no inherent HSC defect in the absence of BM injury, corroborating reports that indicate a lack of involvement of Notch signaling in adult HSC expansion. ${ }^{4,45,47}$ However, chemotherapy severely impaired the generation of HSC in Notch1 $1^{+\Delta T A D}$ mice suggesting that Notch signaling is required for HSC self-renewal. Transplantation of Notch1 $1^{+\Delta T A D}$ HSC reconstituted irradiated WT hosts which showed no pancytopenic symptoms after chemotherapy, indicating that there is no cell-intrinsic defect in HSC generation due to the presence of the Notch1 $\triangle \mathrm{TAD}$ protein. In fact, the reverse experiment, in which WT HSC were transplanted into Notch1+ATAD recipients had the same severe phenotype as the constitutive

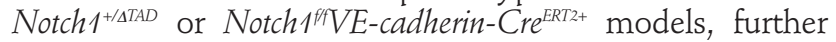
supporting a specific role for Notch signaling in the regeneration of the BM EC niche. Thus, we attribute the loss of HSC and progenitor cells observed after 5-FU treatment to the destruction of the BM EC niche, a process which requires robust Notch activity for its recovery.
Previous work has established that successful T-cell development requires Notch signaling up until $\beta$-selection of $\alpha \beta$ T cells in the thymus. ${ }^{27,5,5,59}$ Notch $1^{+1 / T A D}$ mice exhibited a moderate decrease in developing $T$ cells and had no defect in mature single-positive $\mathrm{T}$ cells (data not shown). This inherent defect was exacerbated by 5 -FU treatment and extended to the BM-residing CLP population. These results suggest that the Notch-driven commitment of the $\mathrm{T}$-cell lineage begins at an early stage in the BM. A recent study, in which genetic deletion of Dll4 in the bone producing mesenchymal Ocn ${ }^{+}$cells decreased CLP numbers and limited the production of T cells in the thymus ${ }^{60}$ supports our observations. Thus, the present findings suggest an important role for Notch1 signaling in mediating the differentiation of early lymphoid hematopoietic progenitors and the existence of a specific Notch-signal-promoting niche in the BM for T-cell commitment prior to thymic migration.

In summary, we employed a novel strategy for physiological inhibition of Notch signaling in adult tissues using a hypomorphic in vivo model system. We demonstrated that high levels of Notch1 signaling are required for the regeneration of $\mathrm{BM} \mathrm{EC}$ after myelosuppressive chemotherapy and radiotherapy. Optimal recuperation of the EC niche relies on several signaling pathways, but it is ultimately a Notch-dependent process that supports regeneration of the BM niche and hematopoietic recovery.

\section{Acknowledgments}

We thank Dr. Warren Pear for invaluable advice and for sharing the Notch $1^{+\Delta T A D}$ murine model system. We also thank Dr. Kishore Wary for sharing the Cdh5-CreERT2 mouse model. Drs. Jon Aster and Stephen Blacklow for advice and thoughtful discussion, Dr. Dawson Gerhardt for her help in generating the Notch1$\triangle T A D$ plasmids and vector constructs, Dr. Jan Kitejewski for helpful advice on Notch mutant mice and Drs. Fotini Gounari and Linda Dagenstein of the University of Chicago transgenic mouse facility for help in maintaining the transgenic mouse colonies. The following cores at the University of Illinois at Chicago contributed to this study: RRC Histology Core and RRC Flow Cytometry Core. This study was funded by NIH grants 1R01HL134971 to KVP and 1R01HL136529 to DL.

\section{References}

1. Li X, Slayton WB. Molecular mechanisms of platelet and stem cell rebound after 5-fluorouracil treatment. Exp Hematol. 2013;41(7):635-645 e633.

2. Kopp HG, Avecilla ST, Hooper AT, et al. Tie2 activation contributes to hemangiogenic regeneration after myelosuppression. Blood. 2005;106(2):505-513.

3. Soligo DA, Lambertenghi Deliliers G, Servida F, et al. Haematopoietic abnormalities after autologous stem cell transplantation in lymphoma patients. Bone Marrow Transplant. 1998;21(1):15-22.

4. Wittels B. Bone marrow biopsy changes following chemotherapy for acute leukemia. Am J Surg Pathol. 1980;4(2):135-142.

5. Hooper AT, Butler JM, Nolan DJ, et al. Engraftment and reconstitution of hematopoiesis is dependent on VEGFR2- mediated regeneration of sinusoidal endothelial cells. Cell Stem Cell. 2009;4 (3):263-274.

6. Islam A. Pattern of bone marrow regeneration following chemotherapy for acute myeloid leukemia. J Med. 1987;18(2):108122.

7. Ding L, Saunders TL, Enikolopov G, Morrison SJ. Endothelial and perivascular cells maintain haematopoietic stem cells. Nature. 2012;481(7382):457-462.

8. Doan PL, Himburg HA, Helms K, et al. Epidermal growth factor regulates hematopoietic regeneration after radiation injury. Nat Med. 2013;19(3):295-304.

9. Arai F, Hirao A, Ohmura M, et al. Tie2/angiopoietin-1 signaling regulates hematopoietic stem cell quiescence in the bone marrow niche. Cell. 2004;118(2):149161.

10. Zhou BO, Ding L, Morrison SJ. Hematopoietic stem and progenitor cells regulate the regeneration of their niche by secreting Angiopoietin-1. Elife. 2015;4: e05521.

11. Doan PL, Russell JL, Himburg HA, et al. Tie2(+) bone marrow endothelial cells regulate hematopoietic stem cell regeneration following radiation injury. Stem Cells. 2013;31(2):327-337.

12. Artavanis-Tsakonas S, Rand MD, Lake RJ. Notch signaling: cell fate control and signal integration in development. Science. 1999;284(5415):770-776.

13. Phng LK, Gerhardt H. Angiogenesis: a team effort coordinated by notch. Dev Cell. 2009;16(2):196-208.

14. Hellstrom M, Phng LK, Hofmann JJ, et al. Dll4 signalling through Notch1 regulates formation of tip cells during angiogenesis. Nature. 2007;445(7129):776-780.

15. Pitulescu ME, Schmidt I, Giaimo BD, et al Dll4 and Notch signalling couples sprouting angiogenesis and artery formation. Nat Cell 
Biol. 2017;19(8):915-927.

16. Briot A, Civelek M, Seki A, et al. Endothelial NOTCH1 is suppressed by circulating lipids and antagonizes inflammation during atherosclerosis. J Exp Med. 2015;212(12):21472163.

17. Mack JJ, Mosqueiro TS, Archer BJ, et al. NOTCH1 is a mechanosensor in adult arteries. Nat Commun. 2017:8(1):1620.

18. Andersson ER, Sandberg R, Lendahl U. Notch signaling: simplicity in design, versatility in function. Development. 2011;138 (17):3593-3612.

19. Kurooka H, Honjo T. Functional interaction between the mouse notch1 intracellular region and histone acetyltransferases PCAF and GCN5. J Biol Chem. 2000;275(22): 17211-17220.

20. Kurooka H, Kuroda K, Honjo T. Roles of the ankyrin repeats and C-terminal region of the mouse notch1 intracellular region. Nucleic Acids Res. 1998;26(23):5448-5455.

21. Ong CT, Cheng HT, Chang LW, et al. Target selectivity of vertebrate notch proteins. Collaboration between discrete domains and CSL-binding site architecture determines activation probability. J Biol Chem. 2006;281(8):5106-5119

22. Gerhardt DM, Pajcini KV, D'Altri T, et al. The Notch1 transcriptional activation domain is required for development and reveals a novel role for Notch1 signaling in fetal hematopoietic stem cells. Genes Dev. 2014;28(6):576-593.

23. High F, Epstein JA. Signalling pathways regulating cardiac neural crest migration and differentiation. Novartis Found Symp. 2007;283:152-161; discussion 161-154, 238241.

24. High FA, Jain R, Stoller JZ, et al. Murine Jagged1/Notch signaling in the second heart field orchestrates Fgf8 expression and tissuetissue interactions during outflow tract development. J Clin Invest. 2009;119(7): 1986-1996.

25. Longley DB, Harkin DP, Johnston PG. 5-fluorouracil: mechanisms of action and clinical strategies. Nat Rev Cancer. 2003;3(5):330338.

26. Lerner C, Harrison DE. 5-Fluorouracil spares hemopoietic stem cells responsible for longterm repopulation. Exp Hematol. 1990;18 (2):114-118.

27. Radtke F, Wilson A, Stark G, et al. Deficient $T$ cell fate specification in mice with an induced inactivation of Notch1. Immunity. 1999;10(5):547-558.

28. Tanigaki K, Han H, Yamamoto N, et al. Notch-RBP-J signaling is involved in cell fate determination of marginal zone B cells. Nat Immunol. 2002;3(5):443-450.

29. Pajcini KV, Xu L, Shao L, et al. MAFB enhances oncogenic Notch signaling in $\mathrm{T}$ cell acute lymphoblastic leukemia. Sci Signal. 2017;10(505).

30. Schepers K, Pietras EM, Reynaud D, et al. Myeloproliferative neoplasia remodels the endosteal bone marrow niche into a selfreinforcing leukemic niche. Cell Stem Cell. 2013;13(3):285-299.

31. Avecilla ST, Hattori K, Heissig B, et al.
Chemokine-mediated interaction of hematopoietic progenitors with the bone marrow vascular niche is required for thrombopoiesis. Nat Med. 2004;10(1):64-71.

32. Kiel MJ, Yilmaz OH, Iwashita T, et al. SLAM family receptors distinguish hematopoietic stem and progenitor cells and reveal endothelial niches for stem cells. Cell. 2005;121(7):1109-1121.

33. Rafii S, Shapiro F, Pettengell R, et al. Human bone marrow microvascular endothelial cells support long-term proliferation and differentiation of myeloid and megakaryocytic progenitors. Blood. 1995;86(9):3353-3363.

34. Shao L, Sun Y, Zhang Z, et al. Deletion of proapoptotic Puma selectively protects hematopoietic stem and progenitor cells against high-dose radiation. Blood. 2010;115(23):4707-4714.

35. Zhao M, Perry JM, Marshall H, et al. Megakaryocytes maintain homeostatic quiescence and promote post-injury regeneration of hematopoietic stem cells. Nat Med. 2014:20(11):1321-1326.

36. Mendelson A, Frenette PS. Hematopoietic stem cell niche maintenance during homeostasis and regeneration. Nat Med 2014;20(8):833-846

37. Yashiro-Ohtani Y, Wang H, Zang C, et al. Long-range enhancer activity determines Myc sensitivity to Notch inhibitors in T cell leukemia. Proc Natl Acad Sci U S A. 2014; 111(46):E4946-4953.

38. Wang H, Zou J, Zhao B, et al. Genome-wide analysis reveals conserved and divergent features of Notch1/RBPJ binding in human and murine T-lymphoblastic leukemia cells Proc Natl Acad Sci U S A. 2011;108(36): 14908-14913.

39. Hassanshahi M, Hassanshahi A, Khabbazi S Su YW, Xian CJ. Bone marrow sinusoidal endothelium: damage and potential regeneration following cancer radiotherapy or chemotherapy. Angiogenesis. 2017;20(4): 427-442.

40. Zhang J, Fukuhara S, Sako $\mathrm{K}$, et al Angiopoietin-1/Tie2 signal augments basal Notch signal controlling vascular quiescence by inducing delta-like 4 expression through AKT-mediated activation of beta-catenin. J Biol Chem. 2011;286(10):8055-8066.

41. Bourillot PY, Aksoy I, Schreiber V, et al. Novel STAT3 target genes exert distinct roles in the inhibition of mesoderm and endoderm differentiation in cooperation with Nanog. Stem Cells. 2009;27(8):17601771.

42. Kumano K, Chiba S, Kunisato A, et al. Notch1 but not Notch2 is essential for generating hematopoietic stem cells from endothelial cells. Immunity. 2003;18(5):699711.

43. Karanu FN, Murdoch B, Gallacher L, et al. The notch ligand jagged-1 represents a novel growth factor of human hematopoietic stem cells. J Exp Med. 2000;192(9):1365-1372.

44. Maillard I, Koch U, Dumortier A, et al. Canonical notch signaling is dispensable for the maintenance of adult hematopoietic stem cells. Cell Stem Cell. 2008;2(4):356366.
45. Mancini SJ, Mantei N, Dumortier A, et al Jagged1-dependent Notch signaling is dispensable for hematopoietic stem cell selfrenewal and differentiation. Blood. 2005;105(6):2340-2342.

46. Poulos MG, Guo P, Kofler NM, et al Endothelial Jagged-1 is necessary for homeostatic and regenerative hematopoiesis. Cell Rep. 2013:4(5):1022-1034.

47. Duarte S, Woll PS, Buza-Vidas N, et al Canonical Notch signaling is dispensable for adult steady-state and stress myelo-erythropoiesis. Blood. 2018;131(15):1712-1719.

48. Kusumbe AP, Ramasamy SK, Itkin T, et al. Age-dependent modulation of vascular niches for haematopoietic stem cells. Nature. 2016;532(7599):380-384.

49. Guo P, Poulos MG, Palikuqi B, et al Endothelial jagged-2 sustains hematopoietic stem and progenitor reconstitution after myelosuppression. J Clin Invest. 2017;127 (12):4242-4256

50. Shirota T, Tavassoli M. Cyclophosphamideinduced alterations of bone marrow endothelium: implications in homing of marrow cells after transplantation. Exp Hematol. 1991;19(5):369-373.

51. Murata K, Hattori M, Hirai N, et al. Hes1 directly controls cell proliferation through the transcriptional repression of p27Kip1. Mol Cell Biol. 2005;25(10):4262-4271.

52. Stine ZE, Walton ZE, Altman BJ, Hsieh AL, Dang CV. MYC, Metabolism, and cancer. Cancer Discov. 2015;5(10):1024-1039.

53. Gale NW, Thurston G, Hackett SF, et al Angiopoietin-2 is required for postnatal angiogenesis and lymphatic patterning, and only the latter role is rescued by Angiopoietin-1. Dev Cell. 2002;3(3):411423.

54. Yancopoulos GD, Davis S, Gale NW, et al Vascular-specific growth factors and blood vessel formation. Nature. 2000;407(6801): 242-248.

55. Juffer P, Jaspers RT, Lips P, Bakker AD, KleinNulend J. Expression of muscle anabolic and metabolic factors in mechanically loaded MLO-Y4 osteocytes. Am J Physiol Endocrinol Metab. 2012;302(4):E389-395.

56. Schlaeppi JM, Gutzwiller S, Finkenzeller G Fournier B. 1,25-Dihydroxyvitamin D3 induces the expression of vascular endothelial growth factor in osteoblastic cells. Endocr Res. 1997;23(3):213-229.

57. Sacchetti B, Funari A, Michienzi S, et al. Selfrenewing osteoprogenitors in bone marrow sinusoids can organize a hematopoietic microenvironment. Cell. 2007;131(2):324336.

58. MacDonald HR, Wilson A, Radtke F. Notch1 and T-cell development: insights from conditional knockout mice. Trends Immunol. 2001:22(3):155-160

59. Wilson A, MacDonald HR, Radtke F. Notch 1-deficient common lymphoid precursors adopt a B cell fate in the thymus. J Exp Med. 2001;194(7):1003-1012.

60. Yu VW, Saez B, Cook C, et al. Specific bone cells produce DLL4 to generate thymusseeding progenitors from bone marrow. J Exp Med. 2015;212(5):759-774. 